\title{
Frontière du fractal de Rauzy et système de numération complexe
}

\author{
par
}

Ali Messaoudi (Marseille)

1. Introduction. On considère le polynôme $P(x)=x^{3}-x^{2}-x-1$. Ce polynôme a une racine réelle $\beta$ strictement supérieure à 1 et deux racines complexes conjuguées $\alpha$ et $\bar{\alpha}$ de module inférieur strictement à 1 .

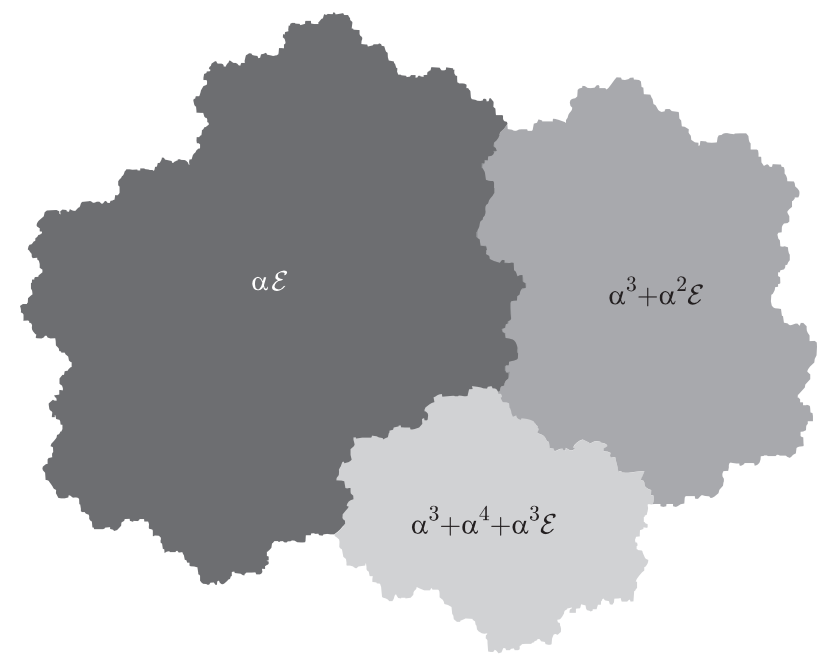

Fig. 1. Le fractal de Rauzy

Le fractal de Rauzy (fig. 1) est l'ensemble

$$
\mathcal{E}=\left\{\sum_{i=3}^{\infty} \varepsilon_{i} \alpha^{i} \mid \forall i \geq 3, \varepsilon_{i} \in\{0,1\}, \varepsilon_{i} \varepsilon_{i+1} \varepsilon_{i+2}=0\right\} .
$$

Il a été introduit par G. Rauzy [17] dans le but de donner une représentation géométrique du système dynamique symbolique associé à la substitution

2000 Mathematics Subject Classification: 28A78, 28A80, 11B39, 11B85, 11K16. 
$\sigma$ définie par

$$
\sigma(0)=01, \quad \sigma(1)=02, \quad \sigma(2)=0 .
$$

Le fractal de Rauzy a plusieurs propriétés : c'est un compact de $\mathbb{C}$, connexe, à frontiére fractale et à intérieur simplement connexe et il induit un pavage périodique de $\mathbb{C}$ modulo $\mathbb{Z}+\mathbb{Z} \alpha$ (voir [15]). Il est partagé en trois régions similaires qui induisent un autre pavage non périodique et auto-similaire du plan complexe. Ces régions sont : $\alpha \mathcal{E}, \alpha^{3}+\alpha^{2} \mathcal{E}$ et $\alpha^{3}+\alpha^{4}+\alpha^{3} \mathcal{E}$.

Le fractal de Rauzy a fait l'objet de plusieures études (voir [1], [17], [10], [15], [19], [11]) et peut être relié à différents problèmes :

- Système de numération complexe [15], [16].

- Représentation géométrique des systèmes dynamiques symboliques [17], [10], [15], [19], [11].

- Méthode de Dekking pour la construction d'objets fractals [10].

- Fractions continues de dimension deux.

- Pavages quasi-périodiques du plan [10], [11]. [15].

- Partitions de Markov pour les automorphismes hyperboliques du tore $\mathbb{T}^{3}$ [1],

La frontière du fractal de Rauzy a été au début étudiée par S. Ito et M. Kimura [10]. Ils ont montré que c'est une courbe de Jordan engendrée par la méthode de Dekking (voir [5]) pour la construction d'objets fractals. Ensuite, en liant la frontière de $\mathcal{E}$ aux nombres complexes qui ont plusieurs développements en base $\alpha$ avec des chiffres dans $\{0,1\}$ sans trois " 1 " consécutifs, il a été construit dans [16] un automate fini qui génére cette frontière.

Dans ce papier nous donnons une paramétrisation de la frontière du fractal de Rauzy. Cela permet de calculer sa dimension de Hausdorff et de montrer que c'est un quasi-cercle. Ensuite nous donnons une méthode de construction des points strictement extrémaux du fractal de Rauzy. Les développements de ces points en base $\alpha$ sont liés au codage d'une rotation d'angle irrationnel donné en fonction de l'argument de $\alpha$ sur le tore $S^{1}$ sous la partition ([0,1/2[, [1/2,1[) ou bien (] $0,1 / 2],] 1 / 2,1])$. Cette construction permet de trouver l'enveloppe convexe du fractal de Rauzy et se généralise aux $k$-fractals du dragon, $k \geq 1$, c'est-à-dire aux ensembles $\mathcal{D}_{k}=\left\{\sum_{n=1}^{\infty} a_{n} /(-k+i)^{n} \mid a_{n} \in\left\{0,1, \ldots, k^{2}\right\}\right\}$ (pour l'étude de ces ensembles, voir [6], [7], [8]).

Dans le cas où $k=1$, c'est-à-dire le fractal du dragon, on montre que l'enveloppe convexe est un octogone, on retrouve ainsi d'une autre façon un résultat de Benedek et Panzone (voir [3]). Ce qui est nouveau avec notre méthode est qu'elle permet de trouver tous les points strictement extrémaux du fractal du dragon et qu'elle se généralise aux ensembles de la forme $\left\{\sum_{i=0}^{\infty} a_{i} \gamma^{i} \mid\left(a_{i}\right) \in A^{\mathbb{N}}\right\}$ où $A$ est un ensemble 
fini de réels positifs contenant 0 , et $\gamma$ un nombre complexe de module $<1$.

REMARQUE. Les résultats que l'on obtiendra sont indépendants du fait que la partie imaginaire de $\alpha$ soit positive ou négative. Les figures du fractal de Rauzy données dans cet article sont faites pour $\alpha$ ayant une partie imaginaire négative, c'est-à-dire $\alpha \sim-0.419-0.606 i$.

2. Notations et définitions. Notons $\mathcal{N}$ l'ensemble des suites $\left(a_{n}\right)_{n \in \mathbb{Z}}$ appartenant à $\{0,1\}^{\mathbb{Z}}$ dans lesquelles ne figurent pas trois " 1 " consécutifs, et telles qu'il existe un entier $k \in \mathbb{Z}$, tel que pour tout entier $n \leq k, a_{n}=0$.

Nous parlerons indifféremment d'une suite $\left(a_{n}\right)_{n \in \mathbb{Z}}$ appartenant à $\mathcal{N}$ telle que $a_{n}=0$ pour tout $n \leq k$ et de la suite $\left(a_{n}\right)_{n \geq k}$. Soit $\left(a_{n}\right)_{n \geq k}$ un élément de $\mathcal{N}$. Supposons qu'il existe $p \in \mathbb{Z}$ tel que pour tout $n \geq p, a_{n}=0$. Cette suite sera notée $\left(a_{n}\right)_{k \leq n \leq p}=a_{k} \ldots a_{p}$ et l'ensemble de telles suites, $\mathcal{N}_{\mathrm{f}}$.

Soit $z \in \mathbb{C}$ et $A \subset \mathbb{C}$. Nous posons $A+z=\{x+z \mid x \in A\}$ et $z A=\{z x \mid x \in A\}$. Nous notons $\operatorname{int}(A)$ l'intérieur de $A, \operatorname{Fr}(A)$ la frontière de $A, \operatorname{diam}(A)$ le diamètre de $A$ et $\bar{A}$ l'adhérence de $A$.

Soit $x$ un réel. Nous notons $[x]$ sa partie entière, $x[1]$ sa partie fractionnaire $x-[x]$. Nous appelons $(\cdot) \bmod 2$ l'application de $\mathbb{Z}$ dans $\{0,1\}$ qui à un entier $n$ associe $n \bmod 2=0$ si $n$ est pair, et 1 sinon.

Un automate fini est la donnée de $(S, A, C)$ où $A$ est l'alphabet de l'automate, $S$ l'ensemble des états, et $C$ un sous-ensemble de $S \times S \times A$.

On ajoute souvent à l'automate un ensemble $I$ d'états initiaux et un ensemble $F$ d'états finaux. Dans cet article, on aura besoin seulement de l'ensemble $I$. On dit qu'une suite $\left(a_{n}\right)$ est reconnaissable par l'automate $(S, A, C)$ s'il existe une suite $\left(s_{n}\right) \in A^{\mathbb{N}}$ telle que $\left(s_{i-1}, s_{i}, a_{i}\right) \in C$ pour tout $i \in \mathbb{N}$.

Soit $(X, f)$ un système dynamique et $\mathcal{P}=\left\{X_{1}, \ldots, X_{k}\right\}, k \in \mathbb{N}$, une partition de $X$. Soit l'alphabet $B=\left\{a_{1}, \ldots, a_{k}\right\}$ et $f_{\mathcal{P}}$ l'application de $X$ dans $B^{\mathbb{N}}$ qui à un élément $x$ de $X$ associe $f_{\mathcal{P}}(x)=\left(v_{n}\right)_{n \in \mathbb{N}}$ où $v_{i}=a_{j}$ si $f^{(i)}(x) \in X_{j}$. La suite $f_{\mathcal{P}}(x)$ est appelée codage de $x$ associé à la partition $\mathcal{P}$ sous l'application $f$.

3. Propriétés de la frontière de $\mathcal{E}$. La frontière de $\mathcal{E}$ est l'union de six $\operatorname{arcs}(\operatorname{voir}[15],[16])$ de la forme $\mathcal{E} \cap(\mathcal{E}+u)$ où $u \in\{1, \alpha, 1+\alpha,-1,-\alpha,-1-\alpha\}$ (fig. 2). En plus si $u \in(\mathbb{Z}+\mathbb{Z} \alpha)-\{0\}$ alors $\mathcal{E} \cap(\mathcal{E}+u) \neq \emptyset$ si et seulement si $u \in\{1, \alpha, 1+\alpha,-1,-\alpha,-1-\alpha\}$. Par ailleurs, il est connu [15] que tout nombre complexe $z$ s'écrit en base $\alpha$ comme

$$
z=\sum_{i=l}^{\infty} \varepsilon_{i} \alpha^{i}, \quad \text { où } l \in \mathbb{Z} \text { et }\left(\varepsilon_{i}\right)_{i \geq l} \in \mathcal{N} \text {. }
$$




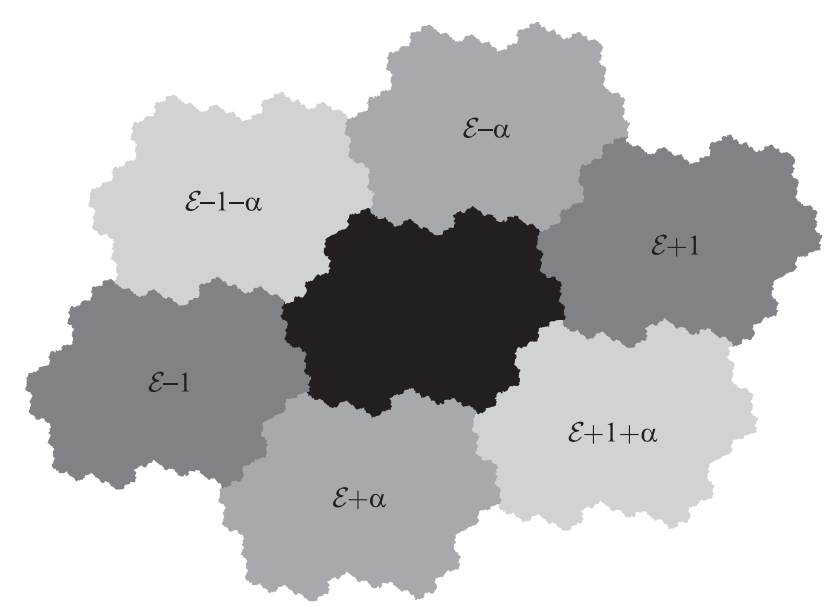

Fig. 2. Pavage périodique du plan par le fractal de Rauzy

La suite $\left(\varepsilon_{i}\right)_{i \geq l}$ sera appelée un $\alpha$-développement de $z$. Un point de la frontière de $\mathcal{E}$ a au moins deux $\alpha$-développements, un $\alpha$-développement provient de $\mathcal{E}$ et l'autre de $\mathcal{E}+u$ où $u \in\{1, \alpha, 1+\alpha,-1,-\alpha,-1-\alpha\}$ (voir [15]). D'autre part, un nombre complexe $z$ ayant au moins deux $\alpha$-développements distincts peut s'écrire comme $z=\sum_{i=k}^{L} a_{i} \alpha^{i}+\alpha^{N} x$ où $\left(a_{i}\right) \in \mathcal{N}_{\mathrm{f}}$ est le début commun des deux $\alpha$ développements de $z$ et $N$ entier relatif choisi de telle manière que $x \in \mathcal{E} \cap(\mathcal{E}+v)$ où $v \in\left\{1, \alpha, \alpha^{2}, 1+\alpha, 1+\alpha^{2}, \alpha+\alpha^{2}\right\}$. D'où $x \in \operatorname{Fr}(\mathcal{E})$. Par conséquent, le problème de la frontière de $\mathcal{E}$ est équivalent au problème des nombres complexes ayant plusieurs $\alpha$-développements.

Ces nombres complexes sont caractérisés par un automate noté $\mathcal{B}$ (fig. 3 ) et nous avons le théorème suivant ([16], p. 145).

ThÉORÈme 1. Soient $\left(a_{i}\right)_{i \geq-L}$ et $\left(b_{i}\right)_{i \geq-L}$ deux éléments distincts de $\mathcal{N}$. Alors

$$
\sum_{i=-L}^{\infty} a_{i} \alpha^{i}=\sum_{i=-L}^{\infty} b_{i} \alpha^{i}
$$

si et seulement si la suite $\left(\left(a_{i}, b_{i}\right)\right)_{i \geq-L}$ est reconnaissable par l'automate $\mathcal{B}$.

L'idée de le construction de l'automate $\mathcal{B}$ (donnée dans [16]) est la suivante :

Soient $x=\sum_{i=-L}^{\infty} a_{i} \alpha^{i}$ et $y=\sum_{i=-L}^{\infty} b_{i} \alpha^{i}$. Nous avons ([16], théorème 1) $x=y$ si et seulement si pour tout $k \geq-L$,

$$
x(k)-y(k) \in S=\left\{0, \pm 1, \pm \alpha, \pm(1+\alpha), \pm\left(1+\alpha^{2}\right), \pm\left(\alpha+\alpha^{2}\right), \pm \alpha^{2}\right\},
$$

où $x(k)=\alpha^{-k+2} \sum_{i=-L}^{k} a_{i} \alpha^{i}$ et $y(k)=\alpha^{-k+2} \sum_{i=-L}^{k} b_{i} \alpha^{i}$. 


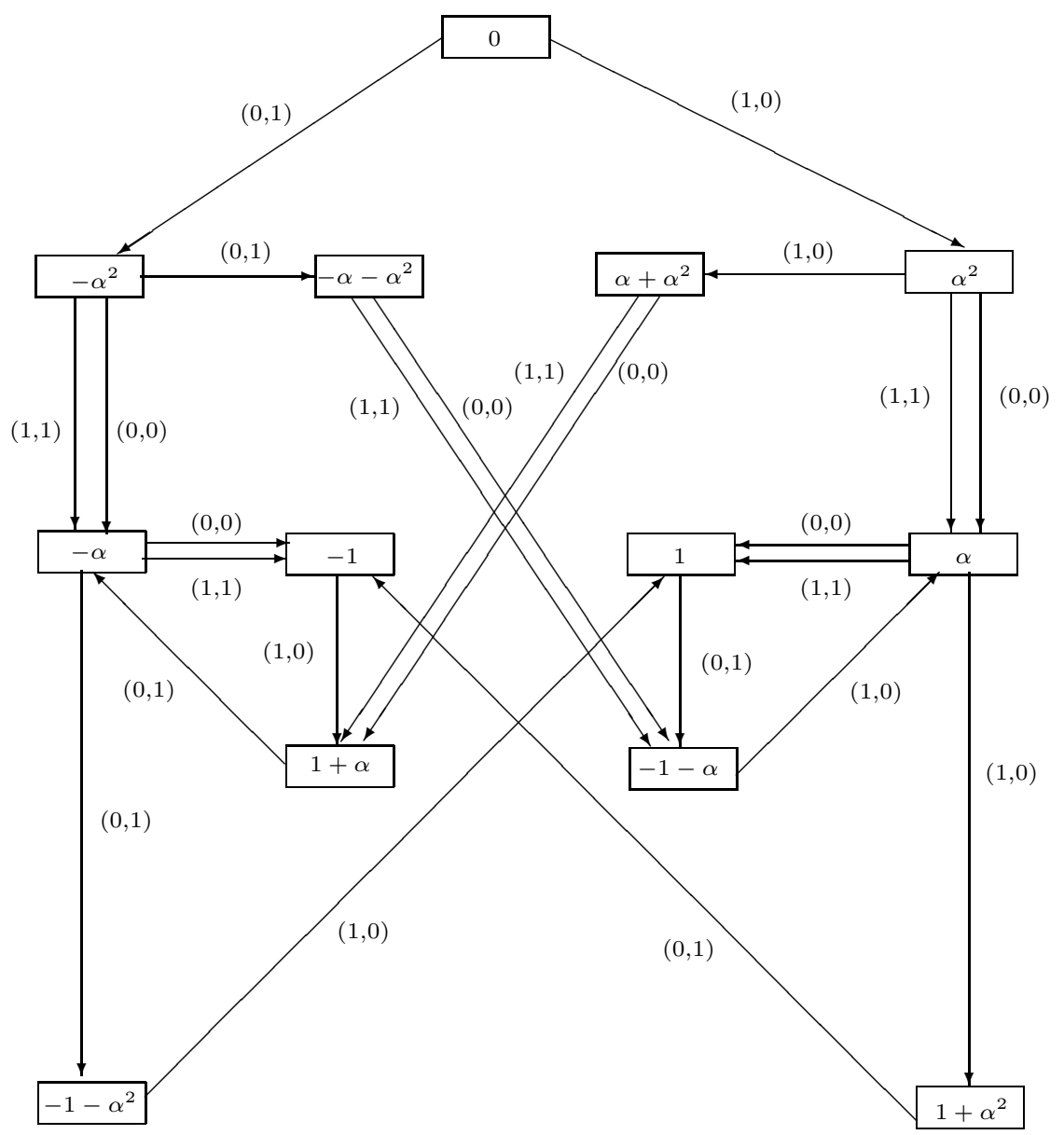

Fig. 3. Automate $\mathcal{B}$

Posons pour tout $k \geq-L, A_{k}=x(k)-y(k)$. Donc

$$
A_{k+1}=\frac{A_{k}}{\alpha}+\left(a_{k+1}-b_{k+1}\right) \alpha^{2} .
$$

Soit $s$ le plus petit entier tel que $a_{s} \neq b_{s}$. D'où $A_{i}=0$ pour tout $i$ dans $\{-L, \ldots, s-1\}$. Supposons que $\left(a_{s}, b_{s}\right)=(1,0)$. Alors $A_{s}=\alpha^{2}$. Nous avons

$$
A_{s+1}=\alpha+\left(a_{s+1}-b_{s+1}\right) \alpha^{2}= \begin{cases}\alpha+\alpha^{2} & \text { si }\left(a_{s+1}, b_{s+1}\right)=(1,0), \\ \alpha & \text { si }\left(a_{s+1}, b_{s+1}\right)=(0,0) \text { ou }(1,1) .\end{cases}
$$

Nous construisons l'automate $\mathcal{B}$ dont les états sont les éléments de $S$. Soient $V$ et $W$ deux éléments de $S$. Nous mettons une flèche étiquetée par $(x, y) \in\{0,1\}^{2}$ et allant de $V$ à $W$ si et seulement si $W=V / \alpha+(x-y) \alpha^{2}$. Nous prenons 0 pour état initial de l'automate $\mathcal{B}$. C'est l'état où les deux $\alpha$-développements ne sont pas encore distincts. 
A. Messaoudi

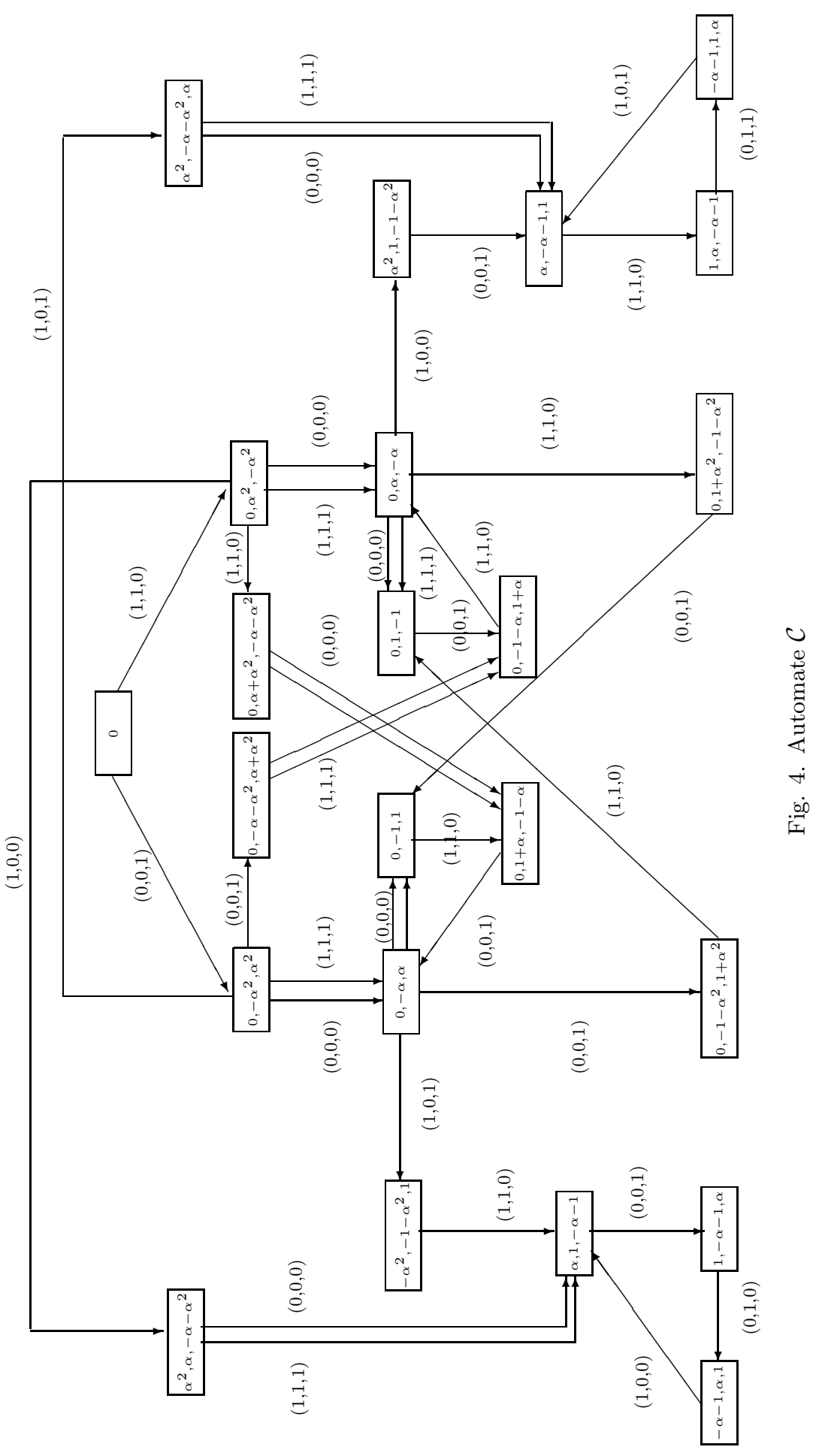


L'état initial est donc lié à l'état $\alpha^{2}$ par une flèche d'étiquette $(1,0)$. L'état $\alpha^{2}$ est lié à l'état $\alpha+\alpha^{2}$ par une flèche d'étiquette $(1,0)$ et à l'état $\alpha$ par deux flèches, une d'étiquette $(0,0)$ et l'autre d'étiquette $(1,1)$. Comme l'ensemble des états $S$ est fini, nous obtenons un automate fini.

De même il existe un automate fini $\mathcal{C}$ (fig. 4) qui reconnait les nombres complexes qui ont trois $\alpha$-développements (voir [16]). Une description detaillée des automates $\mathcal{B}$ et $\mathcal{C}$ se trouve dans [15] et [16].

Remarque. Il est facile de déterminer les points de la frontière de $\mathcal{E}$ à partir de l'automate des nombres complexes doubles, car un point de la frontière a au moins deux $\alpha$-développements : $\left(a_{n}\right)_{n \geq 3}$ et $\left(b_{n}\right)_{n \geq 0}$, tels que $b_{0}+b_{1} \alpha+b_{2} \alpha^{2} \in$ $\left\{1, \alpha, \alpha^{2}, 1+\alpha, 1+\alpha^{2}, \alpha+\alpha^{2}\right\}$.

4. Paramétrisation de la frontière de $\mathcal{E}$. Nous notons les six courbes (fig. 5) constituant la frontière de $\mathcal{E}$ par $X=\mathcal{E} \cap(\mathcal{E}+\alpha), Y=\mathcal{E} \cap(\mathcal{E}+1+\alpha)$, $Z=\mathcal{E} \cap(\mathcal{E}+1), X^{\prime}=\mathcal{E} \cap(\mathcal{E}-\alpha), Y^{\prime}=\mathcal{E} \cap(\mathcal{E}-1-\alpha)$ et $Z^{\prime}=\mathcal{E} \cap(\mathcal{E}-1)$.

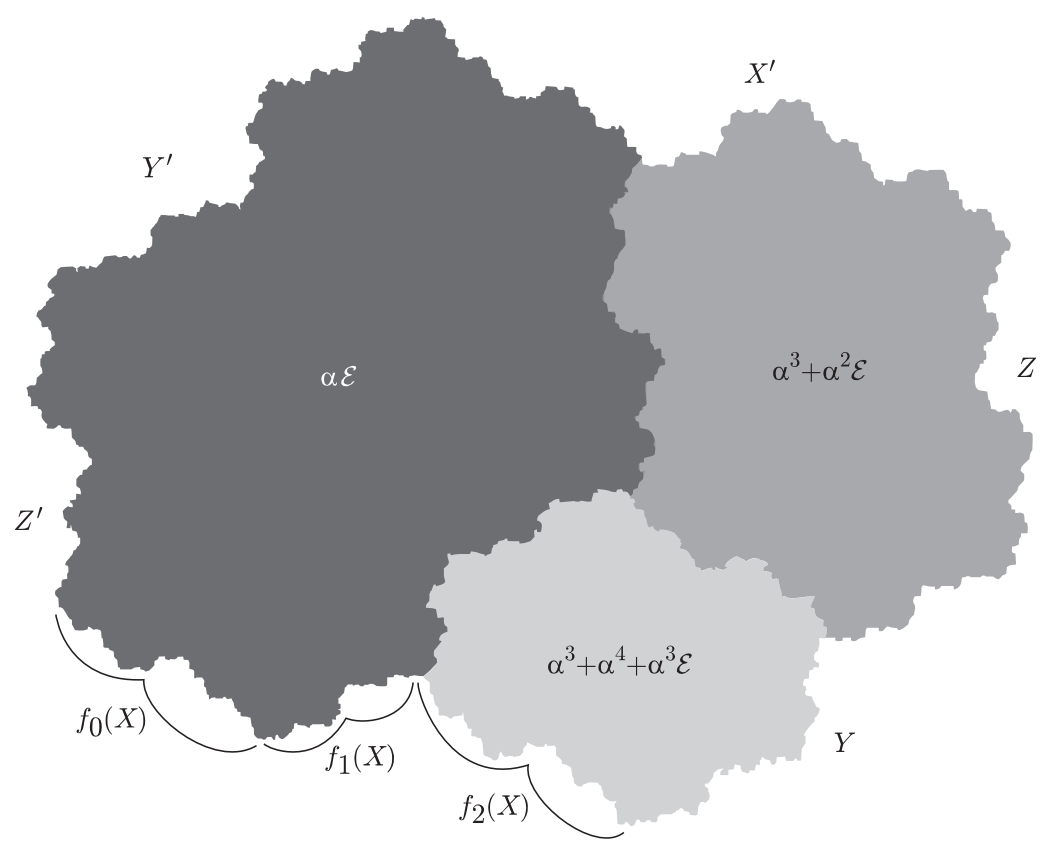

Fig. 5

Dans cette section, nous allons construire une bijection continue entre $[0,1]$ et $X=\mathcal{E} \cap(\mathcal{E}+\alpha)$, ce qui nous permet de calculer la dimension de Hausdorff de la frontière de $\mathcal{E}$, et de montrer que celle-ci est un quasi-cercle. 
Tout d'abord, nous allons montrer que chacune de ses six courbes est l'image d'une autre par une transformation affine; pour cela, nous avons besoin du lemme suivant.

LeMme 1. Les relations suivantes sont vérifiées:

1. $X \cap Y=\left\{-\alpha^{2}\right\}$.

2. $Y \cap Z=\left\{\alpha^{3} /\left(1-\alpha^{3}\right)\right\}$.

3. $Z \cap X^{\prime}=\left\{-\alpha^{2}-\alpha\right\}$.

4. $X^{\prime} \cap Y^{\prime}=\left\{\alpha^{5} /\left(1-\alpha^{3}\right)\right\}$.

5. $Y^{\prime} \cap Z^{\prime}=\left\{-\alpha^{3}\right\}$.

6. $Z^{\prime} \cap X=\left\{\alpha^{4} /\left(1-\alpha^{3}\right)\right\}$.

Preuve. Soit $z$ un élément de $X \cap Y=\mathcal{E} \cap(\mathcal{E}+\alpha) \cap(\mathcal{E}+1+\alpha)$. D'après l'automate $\mathcal{C}$,

$$
z=\sum_{i \geq 1} \alpha^{3 i}+\alpha^{3 i+1}=1+\alpha+\sum_{i \geq 1} \alpha^{3 i+1}+\alpha^{3 i+2}=\alpha+\sum_{i \geq 1} \alpha^{3 i}+\alpha^{3 i+2}=-\alpha^{2} .
$$

De même, l'ensemble $Y \cap Z=\mathcal{E} \cap(\mathcal{E}+1) \cap(\mathcal{E}+1+\alpha)$ est réduit à un singleton $\{x\}$ où

$$
x=\sum_{i \geq 1} \alpha^{3 i}=1+\sum_{i \geq 1} \alpha^{3 i+1}=1+\alpha+\sum_{i \geq 1} \alpha^{3 i+2}=\frac{\alpha^{3}}{1-\alpha^{3}} .
$$

Les autres relations découlent du fait que $Z \cap X^{\prime}=X \cap Y-\alpha, X^{\prime} \cap Y^{\prime}=$ $Y \cap Z-1-\alpha, Y^{\prime} \cap Z^{\prime}=X \cap Y-1-\alpha$ et $Z^{\prime} \cap X=X \cap Y-1$.

Lemme 2. Les propriétés suivantes sont vérifiées:

1. $Y=1+\alpha+\alpha X$.

2. $Z=\alpha^{3}+\alpha^{2} X$.

3. $X^{\prime}=-\alpha+X$.

4. $Y^{\prime}=\alpha X$.

5. $Z^{\prime}=\alpha+\alpha^{2}+\alpha^{2} X$.

Preuve. 1. Soit $z$ un élément de $Y$. En vertu de l'automate $\mathcal{B}$, nous avons trois cas :

- $z=1+\alpha+\alpha^{5}+\alpha^{3} w_{1}=\alpha^{3}+\alpha^{6}+\alpha^{4} w_{1}^{\prime}$ où $w_{1}, w_{1}^{\prime} \in \mathcal{E}$. Dans ce cas

$$
(z-1-\alpha) / \alpha=\alpha^{4}+\alpha^{2} w_{1}=\alpha+\alpha^{5}+\alpha^{3} w_{1}^{\prime} .
$$

- $z=1+\alpha+\alpha^{4}+\alpha^{5}+\alpha^{4} w_{2}=\alpha^{3}+\alpha^{4}+\alpha^{6}+\alpha^{4} w_{2}^{\prime}$ où $w_{2}, w_{2}^{\prime} \in \mathcal{E}$, donc

$$
(z-1-\alpha) / \alpha=\alpha^{3}+\alpha^{4}+\alpha^{3} w_{2}=\alpha+\alpha^{3}+\alpha^{5}+\alpha^{3} w_{2}^{\prime} .
$$

- $z=1+\alpha+\alpha^{5}+\alpha^{7}+\alpha^{5} w_{3}=\alpha^{3}+\alpha^{4}+\alpha^{6}+\alpha^{5} w_{3}^{\prime}$ où $w_{3}, w_{3}^{\prime} \in \mathcal{E}$, d'où

$$
(z-1-\alpha) / \alpha=\alpha^{4}+\alpha^{6}+\alpha^{4} w_{3}=\alpha+\alpha^{3}+\alpha^{5}+\alpha^{4} w_{3}^{\prime} .
$$

Par conséquent $(z-1-\alpha) / \alpha \in X$. 
Réciproquement, si $z$ appartient à $X$, alors

$$
\alpha z+1+\alpha \in(\alpha \mathcal{E}+1+\alpha) \cap\left(\alpha \mathcal{E}+\alpha^{3}\right) \subset Y
$$

2. Soit $z$ élément de $Z$, donc $z=1+\alpha^{4}+\alpha^{2} w=\alpha^{3}+\alpha^{2} w^{\prime}$ où $w, w^{\prime} \in \mathcal{E}$, d'où

$$
\left(z-\alpha^{3}\right) / \alpha^{2}=\alpha+w=w^{\prime} \in X .
$$

Par ailleurs, $\alpha^{3}+\alpha^{2} X=\left(\alpha^{3}+\alpha^{2} \mathcal{E}\right) \cap\left(2 \alpha^{3}+\alpha^{2} \mathcal{E}\right)$. Comme $2 \alpha^{3}=\alpha^{4}+1$, nous avons $\alpha^{3}+\alpha^{2} X=\left(\alpha^{3}+\alpha^{2} \mathcal{E}\right) \cap\left(1+\alpha^{4}+\alpha^{2} \mathcal{E}\right) \subset Z$. Il en résulte que

$$
Z=\alpha^{3}+\alpha^{2} X
$$

Les autres relations découlent des relations

$$
Y^{\prime}=Y-1-\alpha, \quad Z^{\prime}=Z-1, \quad X^{\prime}=X-\alpha .
$$

D'où le lemme.

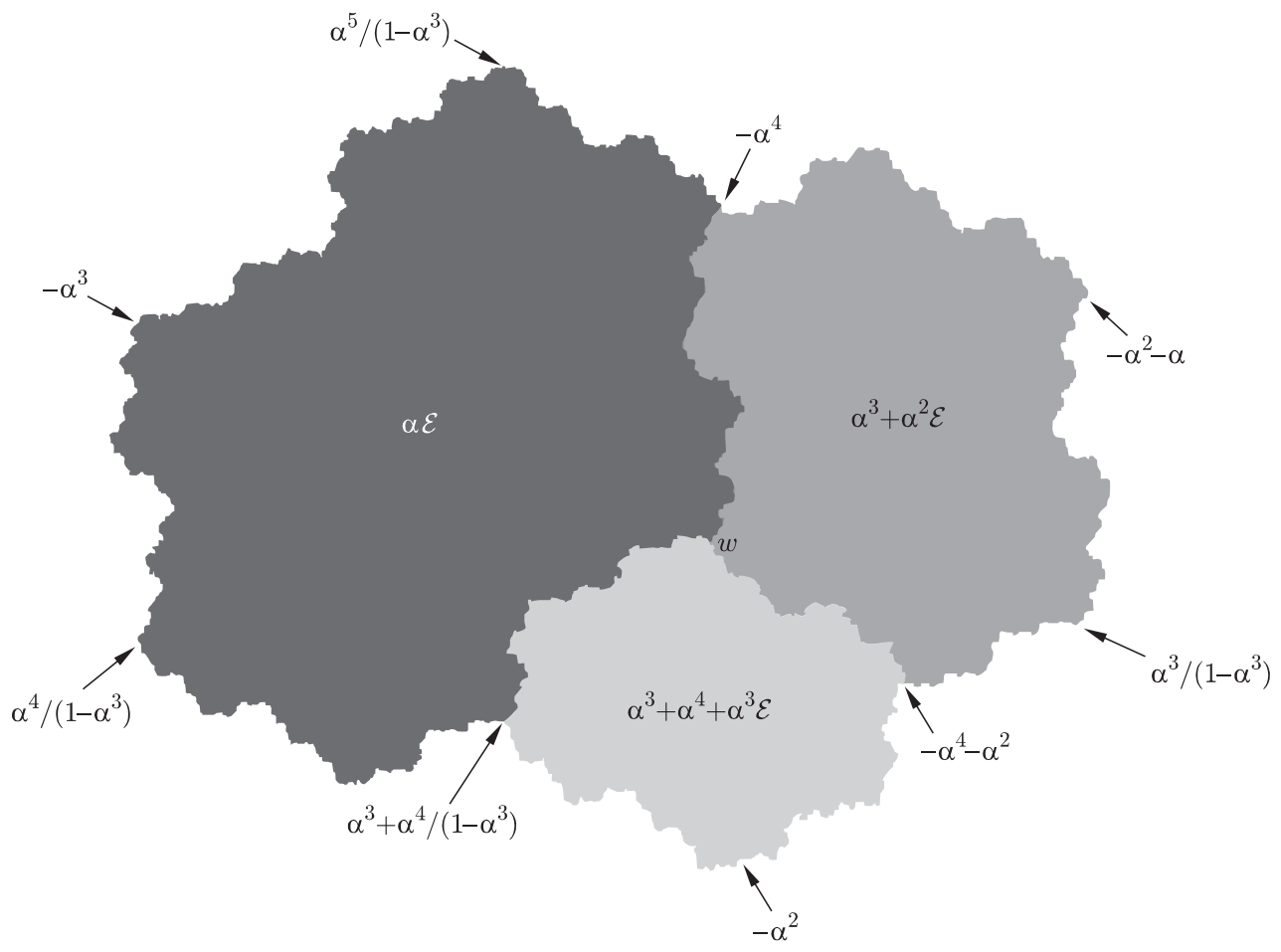

Fig. 6

Maintenant, nous allons étudier l'ensemble $X$. On remarque que $X$ est autoaffine et partagé en trois régions similaires (voir fig. 6). Nous allons montrer que chacune de ses régions correspond à l'image de $X$ par l'une des trois fonctions $g_{i}$, 
$i \in\{0,1,2\}$, définies par :

$\forall z \in \mathbb{C}, \quad g_{0}(z)=\alpha^{4}+\alpha^{3} z, g_{1}(z)=\alpha+\alpha^{3}+\alpha^{5}+\alpha^{4} z, g_{2}(z)=\alpha^{3}+\alpha^{4}+\alpha^{3} z$.

Pour cela, nous nous serverons du lemme suivant.

Lemme 3. $X$ vérifie les propriétés suivantes:

1. $X=g_{0}(X) \cup g_{1}(X) \cup g_{2}(X)$.

2. $g_{0}(X) \cap g_{1}(X)=-\alpha^{3}-\alpha^{2}$.

3. $g_{1}(X) \cap g_{2}(X)=\alpha^{3}+\alpha^{4} /\left(1-\alpha^{3}\right)$.

4. $g_{0}(X) \cap g_{2}(X)=\emptyset$.

Preuve. 1. Puisque $X=\mathcal{E} \cap(\mathcal{E}+\alpha)$, nous avons

$$
\begin{aligned}
& g_{0}(X)=\left(\alpha^{4}+\alpha^{3} \mathcal{E}\right) \cap\left(2 \alpha^{4}+\alpha^{3} \mathcal{E}\right)=\left(\alpha^{4}+\alpha^{3} \mathcal{E}\right) \cap\left(\alpha+\alpha^{5}+\alpha^{3} \mathcal{E}\right), \\
& g_{1}(X)=\left(\alpha+\alpha^{3}+\alpha^{5}+\alpha^{4} \mathcal{E}\right) \cap\left(\alpha^{4}+\alpha^{6}+\alpha^{4} \mathcal{E}\right), \\
& g_{2}(X)=\left(\alpha^{3}+\alpha^{4}+\alpha^{3} \mathcal{E}\right) \cap\left(\alpha+\alpha^{3}+\alpha^{5}+\alpha^{3} \mathcal{E}\right)
\end{aligned}
$$

Donc, pour tout $i$ élément de $\{0,1,2\}, g_{i}(X)$ est inclus dans $X$.

Soit $z$ un élément de $X$. En vertu de l'automate $\mathcal{B}$, nous avons trois cas :

- $z=\alpha+\alpha^{5}+\alpha^{3} w_{0}=\alpha^{4}+\alpha^{3} w_{0}^{\prime}$ où $w_{0}, w_{0}^{\prime} \in \mathcal{E}$. Dans ce cas,

$$
g_{0}^{-1}(z)=\alpha+w_{0}=w_{0}^{\prime} \in X, \quad \text { d'où } \quad z \in g_{0}(X) .
$$

- $z=\alpha+\alpha^{3}+\alpha^{5}+\alpha^{4} w_{1}=\alpha^{4}+\alpha^{6}+\alpha^{4} w_{1}^{\prime}$ où $w_{1}, w_{1}^{\prime} \in \mathcal{E}$. Donc

$$
g_{1}^{-1}(z)=w_{1}=\alpha+w_{1}^{\prime} \in X, \quad \text { d'où } \quad z \in g_{1}(X) .
$$

- $z=\alpha+\alpha^{3}+\alpha^{5}+\alpha^{3} w_{2}=\alpha^{3}+\alpha^{4}+\alpha^{3} w_{2}^{\prime}$ où $w_{2}, w_{2}^{\prime} \in \mathcal{E}$, alors

$$
g_{2}^{-1}(z)=\alpha+w_{2}=w_{2}^{\prime} \in X, \quad \text { d'où } \quad z \in g_{2}(X) .
$$

Par conséquent $X=g_{0}(X) \cup g_{1}(X) \cup g_{2}(X)$.

2. Supposons que $x$ appartient à $g_{0}(X) \cap g_{1}(X)$. Il existe $z$ et $z^{\prime}$ deux éléments de $X$ tels que

$$
x=\alpha^{4}+\alpha^{3} z=\alpha+\alpha^{3}+\alpha^{5}+\alpha^{4} z^{\prime},
$$

d'où $z=1+\alpha+\alpha z^{\prime}$, ce qui implique que

$$
z \in \mathcal{E} \cap(\mathcal{E}+\alpha) \cap(\mathcal{E}+1+\alpha),
$$

d'où $z=-\alpha^{2}$, par conséquent $x=-\alpha^{3}-\alpha^{2}$.

3. Soit $x$ un élément de $g_{1}(X) \cap g_{2}(X)$, donc $x=\alpha^{3}+\alpha^{4}+\alpha^{3} z=\alpha+\alpha^{3}+$ $\alpha^{5}+\alpha^{4} z^{\prime}$. Il s'ensuit que $z$ et $z^{\prime} \in X$ d'où $z=\alpha+\alpha z^{\prime} \in \mathcal{E} \cap(\alpha \mathcal{E}+\alpha)$. Par ailleurs, l'automate $\mathcal{B}$ implique que l'ensemble $\mathcal{E} \cap(\alpha \mathcal{E}+\alpha)$ est inclus dans $\alpha \mathcal{E} \cap(\mathcal{E}+\alpha)$. Par conséquent

$$
z^{\prime}=\frac{z}{\alpha}-1 \in \mathcal{E} \cap(\mathcal{E}+\alpha) \cap(\mathcal{E}-1)=\left\{\frac{\alpha^{4}}{1-\alpha^{3}}\right\},
$$

ce qui entraîne que $x=\alpha^{3}+\alpha^{4} /\left(1-\alpha^{3}\right)$. 
4. Soient $z$ et $z^{\prime}$ deux éléments de $X$ tels que $\alpha^{4}+\alpha^{3} z=\alpha^{3}+\alpha^{4}+\alpha^{3} z^{\prime}$. Alors $z=1+z^{\prime}$, d'où $z \in \mathcal{E} \cap(\mathcal{E}+\alpha) \cap(\mathcal{E}+1)=\emptyset$. Il en découle que $g_{0}(X) \cap g_{2}(X)=\emptyset$.

Ceci achève la preuve.

Paramétrisation de $X$. Soient $a$ et $b$ appartenant à $\operatorname{Fr}(\mathcal{E})$. Notons par $\mathrm{I}(a, b)$ l'arc de $\operatorname{Fr}(\mathcal{E})$ d'origine $a$ et d'extrémité $b$ dans le sens trigonométrique. En vertu du lemme 3 , nous avons

$$
\begin{aligned}
& g_{0}(X)=\mathrm{I}\left(\frac{\alpha^{4}}{1-\alpha^{3}},-\alpha^{3}-\alpha^{2}\right)=\mathrm{I}\left(g_{0}\left(\frac{\alpha^{4}}{1-\alpha^{3}}\right), g_{0}\left(-\alpha^{2}\right)\right), \\
& g_{1}(X)=\mathrm{I}\left(-\alpha^{3}-\alpha^{2}, \alpha^{3}+\frac{\alpha^{4}}{1-\alpha^{3}}\right)=\mathrm{I}\left(g_{1}\left(-\alpha^{2}\right), g_{1}\left(\frac{\alpha^{4}}{1-\alpha^{3}}\right)\right), \\
& g_{2}(X)=\mathrm{I}\left(\alpha^{3}+\frac{\alpha^{4}}{1-\alpha^{3}},-\alpha^{2}\right)=\mathrm{I}\left(g_{2}\left(\frac{\alpha^{4}}{1-\alpha^{3}}\right), g_{2}\left(-\alpha^{2}\right)\right) .
\end{aligned}
$$

Pour paramétriser $X$, nous déterminerons trois fonctions complexes $f_{i}, i \in\{0,1,2\}$, telles que $f_{i}(X)$ soit égal à $\mathrm{I}\left(f_{i}\left(\alpha^{4} /\left(1-\alpha^{3}\right)\right), f_{i}\left(-\alpha^{2}\right)\right)$. Pour cela, nous nous serverons de la symétrie de $X$.

LEMme 4. L'ensemble $X$ a un centre de symétrie $C_{0}=\frac{1}{2}\left(\alpha+\frac{\alpha^{6}}{1-\alpha^{3}}\right)$.

Preuve. Posons $C=\alpha^{6} /\left(1-\alpha^{3}\right)$. Puisque $C=\sum_{i=3}^{\infty} \alpha^{i}$, si $z=\sum_{i=3}^{\infty} a_{i} \alpha^{i} \in$ $\mathcal{E}$ alors

$$
C-z=\sum_{i=3}^{\infty}\left(1-a_{i}\right) \alpha^{i} \in \mathcal{E}
$$

Il en résulte que $C / 2$ est un centre de symétrie de $\mathcal{E}$. Par ailleurs,

$$
2 C_{0}-X=\left(\alpha+\frac{\alpha^{6}}{1-\alpha^{3}}-\mathcal{E}\right) \cap\left(\frac{\alpha^{6}}{1-\alpha^{3}}-\mathcal{E}\right)=(\mathcal{E}+\alpha) \cap \mathcal{E}=X
$$

D'où le lemme.

Notons par $S$ la symétrie centrale de centre $C_{0}, S(z)=2 C_{0}-z$ pour tout $z \in X$, et considérons les trois fonctions complexes $f_{0}, f_{1}$ et $f_{2}$ définies par

$$
\begin{aligned}
& f_{0}(z)=g_{0}(z)=\alpha^{4}+\alpha^{3} z \\
& f_{1}(z)=g_{1}(S z)=\alpha^{4}+\alpha^{6}+\alpha^{10} /\left(1-\alpha^{3}\right)-\alpha^{4} z \\
& f_{2}(z)=g_{2}(z)=\alpha^{3}+\alpha^{4}+\alpha^{3} z
\end{aligned}
$$

Soit $z$ un élément de $X$. Puisque $X=f_{0}(X) \cup f_{1}(X) \cup f_{2}(X)$, il existe $z_{1}$ appartenant à $X$ et $a_{0}$ un élément de $\{0,1,2\}$ tel que $z=f_{a_{0}}\left(z_{1}\right)$. De proche en proche, nous construisons une suite $\left(a_{n}\right)_{n \in \mathbb{N}}$ dans $\{0,1,2\}^{\mathbb{N}}$ et une suite $\left(z_{n}\right)_{n \in \mathbb{N}}$ dans $X$, tels que pour tout entier naturel $n$,

$$
z=f_{a_{0}} \circ f_{a_{1}} \circ \ldots \circ f_{a_{n}}\left(z_{n+1}\right)
$$


Comme les fonctions $f_{i}$ sont contractantes, $f_{a_{0}} \circ f_{a_{1}} \circ \ldots \circ f_{a_{n}}\left(z_{n+1}\right)$ tend vers $z$ quand $n$ tend vers l'infini, et que pour tout $x \in X, f_{a_{0}} \circ f_{a_{1}} \circ \ldots \circ f_{a_{n}}(x)$ tend vers $z$ quand $n$ tend vers l'infini.

Fixons $x_{0} \in X$, et définissons une correspondance $f$ de l'ensemble $[0,1]$ dans $X$ de la manière suivante : Soit $t$ un élément de $[0,1]$. Si $\sum_{i=1}^{\infty} a_{i} 3^{-i}$, $\left(a_{i}\right) \in\{0,1,2\}^{\mathbb{N}}$ est un développement de $t$ en base 3 , alors

$$
f(t)=\lim _{n \rightarrow \infty} f_{a_{1}} \circ f_{a_{2}} \circ \ldots \circ f_{a_{n}}\left(x_{0}\right)
$$

Dans tout ce qui suit, nous supposons que si $t$ et $t^{\prime}$ appartiennent à $[0,1]$, alors $t=\sum_{i=1}^{\infty} a_{i} 3^{-i}$ et $t^{\prime}=\sum_{i=1}^{\infty} b_{i} 3^{-i}$, où $a_{i}$ et $b_{i}$ sont des éléments de $\{0,1,2\}$ tels que $a_{i}=b_{i}$ pour $i<k$ et $a_{k}<b_{k}, k \in \mathbb{N}$.

Proposition 1. La correspondance $f$ est une application bijective, continue et vérifie $f(0)=\alpha^{4} /\left(1-\alpha^{3}\right)$ et $f(1)=-\alpha^{2}$.

Pour la preuve, nous avons besoin des lemmes suivants.

Lemme 5. Soient $t$ et $t^{\prime}$ deux éléments de $[0,1]$. Alors

(1) Si $\left|t-t^{\prime}\right|<3^{-N}$ où $N>k$, alors $b_{k}=a_{k}+1, b_{i}=0$ et $a_{i}=2$ pour tout $i$ vérifiant $k+1 \leq i \leq N$.

(2) Si $t=t^{\prime}$ alors $b_{k}=a_{k}+1, b_{i}=0$ et $a_{i}=2$ pour $i \geq k+1$.

Preuve. (2) est une conséquence immédiate de (1).

(1) provient du fait que

$$
\begin{aligned}
3^{-N} & >\sum_{i=1}^{\infty}\left(b_{i}-a_{i}\right) 3^{-i}=\left(b_{k}-a_{k}\right) 3^{-k}+\sum_{i=k+1}^{\infty}\left(b_{i}-a_{i}\right) 3^{-i} \\
& =\left(b_{k}-a_{k}-1\right) 3^{-k}+\sum_{i=k+1}^{\infty}\left(2+b_{i}-a_{i}\right) 3^{-i} .
\end{aligned}
$$

Ceci achève la preuve.

Lemme 6. Soient $h$ et $k$ deux éléments de $\{0,1,2\}$ tels que $h<k$ et soient $x$ et $y$ deux éléments de $X$. Alors $f_{h}(x)=f_{k}(x)$ si et seulement si $k=h+1, x=-\alpha^{2}$ et $y=\alpha^{4} /\left(1-\alpha^{3}\right)$.

Preuve. L'implication réciproque est évidente. Prouvons l'implication directe.

L'ensemble $f_{0}(X) \cap f_{2}(X)$ étant vide, les entiers $h$ et $k$ sont nécessairement consécutifs. Nous avons donc deux cas à étudier :

- Si $h=0, k=1$, alors $f_{0}(x)=f_{1}(y) \Leftrightarrow g_{0}(x)=g_{1}(S y)$; d'où d'après le lemme 3 , nous avons $g_{0}(x)=g_{1}(S y)=-\alpha^{3}-\alpha^{2}$, ou encore $x=S(y)=-\alpha^{2}$. Il en résulte que

$$
x=-\alpha^{2} \quad \text { et } \quad y=\alpha^{4} /\left(1-\alpha^{3}\right) .
$$


- Si $h=1, k=2$, alors $f_{1}(x)=f_{2}(y) \Leftrightarrow g_{1}(S x)=g_{2}(y)$, donc $S x=y=$ $\alpha^{4} /\left(1-\alpha^{3}\right)$, d'où

$$
x=-\alpha^{2}, \quad y=\alpha^{4} /\left(1-\alpha^{3}\right) .
$$

Lemme 7. $f(t)=\alpha^{4} /\left(1-\alpha^{3}\right)$ si et seulement si $t=0$ et $f(t)=1$ si et seulement si $t=-\alpha^{2}$.

Preuve. Puisque $\alpha^{4} /\left(1-\alpha^{3}\right) \notin f_{1}(X) \cup f_{2}(X)$ et

$$
f(t)=f_{a_{1}} \circ f\left(\sum_{i=2}^{\infty} a_{i} 3^{-i}\right)
$$

nous avons

$$
f(t)=\alpha^{4} /\left(1-\alpha^{3}\right) \Leftrightarrow a_{1}=0 .
$$

Or $\alpha^{4} /\left(1-\alpha^{3}\right)$ est le seul point fixe de $f_{0}$; cela entraîne que $f\left(\sum_{i=2}^{\infty} a_{i} 3^{-i}\right)=$ $\alpha^{4} /\left(1-\alpha^{3}\right)$. En itérant le procédé, nous montrons que pour tout entier naturel $n$ non nul, $a_{n}=0$, d'où $t=0$. En utilisant le même raisonnement et le fait que $-\alpha^{2}$ est le seul point fixe de $f_{2}$, nous prouvons que $f(t)=1$ si et seulement si $t=-\alpha^{2}$.

Preuve de la proposition 1

$f$ est bien définie. Soient $t$ et $t^{\prime}$ dans $[0,1]$. Si $t=t^{\prime}$, alors d'après le lemme 5 , $b_{k}=a_{k}+1, b_{i}=0$ et $a_{i}=2$ pour tout $i \geq k+1$. Donc

$$
\begin{aligned}
f(t) & =f_{a_{1}} \ldots f_{a_{k-1}} f_{a_{k}}\left(\lim _{n \rightarrow \infty} f_{2}^{(n)}\left(x_{0}\right)\right), \\
f\left(t^{\prime}\right) & =f_{a_{1}} \ldots f_{a_{k-1}} f_{a_{k}+1}\left(\lim _{n \rightarrow \infty} f_{0}^{(n)}\left(x_{0}\right)\right) .
\end{aligned}
$$

Or

$$
\lim _{n \rightarrow \infty} f_{0}^{(n)}\left(x_{0}\right)=\lim _{n \rightarrow \infty} f_{0}^{(n)}\left(\frac{\alpha^{4}}{1-\alpha^{3}}\right)=\frac{\alpha^{4}}{1-\alpha^{3}},
$$

car $f_{0}\left(\alpha^{4} /\left(1-\alpha^{3}\right)\right)=\alpha^{4} /\left(1-\alpha^{3}\right)$, de même

$$
\lim _{n \rightarrow \infty} f_{2}^{(n)}\left(x_{0}\right)=\lim _{n \rightarrow \infty} f_{2}^{(n)}\left(-\alpha^{2}\right)=-\alpha^{2},
$$

car $f_{2}\left(-\alpha^{2}\right)=-\alpha^{2}$. Il résulte du lemme 6 que $f(t)=f\left(t^{\prime}\right)$.

$f$ est injective. Nous avons

$$
\begin{aligned}
& f(t)=f_{a_{1}} \ldots f_{a_{k-1}} \circ f\left(\sum_{i=1}^{\infty} a_{i+k-1} 3^{-i}\right), \\
& f\left(t^{\prime}\right)=f_{b_{1}} \ldots f_{b_{k-1}} \circ f\left(\sum_{i=1}^{\infty} b_{i+k-1} 3^{-i}\right) .
\end{aligned}
$$


Comme les fonctions $f_{i}$ sont bijectives (rappelons que $a_{i}=b_{i}$ pour $1 \leq i \leq$ $k-1)$,

$$
f(t)=f\left(t^{\prime}\right) \Leftrightarrow f\left(\sum_{i=1}^{\infty} a_{i+k-1} 3^{-i}\right)=f\left(\sum_{i=1}^{\infty} b_{i+k-1} 3^{-i}\right)
$$

D'où

$$
f_{a_{k}} \circ f\left(3 t_{1}-a_{k}\right)=f_{b_{k}} \circ f\left(3 t_{1}^{\prime}-b_{k}\right),
$$

où $t_{1}=\sum_{i=1}^{\infty} a_{i+k-1} 3^{-i}$ et $t_{1}^{\prime}=\sum_{i=1}^{\infty} b_{i+k-1} 3^{-i}$. Il découle du lemme 6 que $b_{k}=$ $a_{k}+1, f\left(3 t_{1}-a_{k}\right)=-\alpha^{2}$ et $f\left(3 t_{1}^{\prime}-b_{k}\right)=\alpha^{4} /\left(1-\alpha^{3}\right)$. Par conséquent, le lemme 7 implique que

$$
3 t_{1}-a_{k}=1 \quad \text { et } 3 t_{1}^{\prime}-b_{k}=0 .
$$

Or $b_{k}=a_{k+1}$, d'où $t_{1}=t_{1}^{\prime}$ ou encore $t=t^{\prime}$.

$f$ étant surjective par construction, donc $f$ est bijective.

$f$ est continue. Supposons que $0<\left|t-t^{\prime}\right|<3^{-N}, N \in \mathbb{N}, N>k$. Le lemme 5 entraîne que

$$
\left|f(t)-f\left(t^{\prime}\right)\right|=\left|f_{a_{1}} \ldots f_{a_{k-1}} \circ f\left(t_{1}\right)-f_{a_{1}} \ldots f_{a_{k-1}} \circ f\left(t_{1}^{\prime}\right)\right|,
$$

où

$$
f\left(t_{1}\right)=f_{a_{k}} \circ f_{2}^{N-k}\left(z_{1}\right) \quad \text { et } \quad f\left(t_{1}^{\prime}\right)=f_{a_{k}+1} \circ f_{0}^{N-k}\left(z_{1}^{\prime}\right),
$$

où $z_{1}, z_{1}^{\prime} \in X$. Par conséquent,

$$
\left|f(t)-f\left(t^{\prime}\right)\right| \leq\left|f_{a_{k}} \circ f_{2}^{N-k}\left(z_{1}\right)-f_{a_{k}+1} \circ f_{0}^{N-k}\left(z_{1}^{\prime}\right)\right| \cdot|\alpha|^{3(k-1)} .
$$

Puisque $f_{a_{k}} \circ f_{2}^{N-k}\left(-\alpha^{2}\right)=f_{a_{k}+1} \circ f_{0}^{N-k}\left(\alpha^{4} /\left(1-\alpha^{3}\right)\right)$, nous avons

$$
\begin{aligned}
\left|f(t)-f\left(t^{\prime}\right)\right| \leq & \left(\left|f_{a_{k}} \circ f_{2}^{N-k}\left(z_{1}\right)-f_{a_{k}} \circ f_{2}^{N-k}\left(-\alpha^{2}\right)\right|\right. \\
& \left.+\left|f_{a_{k}+1} \circ f_{2}^{N-k}\left(z_{1}^{\prime}\right)-f_{a_{k}+1} \circ f_{0}^{N-k}\left(\frac{\alpha^{4}}{1-\alpha^{3}}\right)\right|\right)|\alpha|^{3(k-1)} \\
\leq & \left(|\alpha|^{3(N-k)+3}+|\alpha|^{3(N-k)+4}\right)|\alpha|^{3(k-1)} \cdot \operatorname{diam}(X) \\
\leq & \operatorname{diam}(X) \cdot(1+|\alpha|)|\alpha|^{3 N} .
\end{aligned}
$$

Corollaire 1. La frontière de $\mathcal{E}$ est une courbe de Jordan.

Proposition 2. L'application $f$ est $\delta=-3 \log (|\alpha|) / \log 3$ Hölder continue.

Preuve. Montrons qu'il existe un réel $C>0$ tel que

$$
\forall t, t^{\prime} \in[0,1], \quad\left|f(t)-f\left(t^{\prime}\right)\right| \leq C\left|t-t^{\prime}\right|^{\delta} .
$$

Soient $t$ et $t^{\prime} \in[0,1]$. Supposons que $3^{-N-1} \leq\left|t-t^{\prime}\right|<3^{-N}$ où $N \in \mathbb{N}$.

- Si $N>k$, alors $\left|f(t)-f\left(t^{\prime}\right)\right| \leq \operatorname{diam}(X) \cdot(1+|\alpha|)|\alpha|^{3 N}$.

- Si $N<k$, alors

$$
\begin{aligned}
\left|f(t)-f\left(t^{\prime}\right)\right| & =\left|f_{a_{1}} \ldots f_{a_{k-1}}\left(z_{1}\right)-f_{a_{1}} \ldots f_{a_{k-1}}\left(z_{1}^{\prime}\right)\right| \leq \operatorname{diam}(X) \cdot|\alpha|^{3(k-1)} \\
& \leq \operatorname{diam}(X) \cdot|\alpha|^{3 N}
\end{aligned}
$$


D'où $\left|f(t)-f\left(t^{\prime}\right)\right| \leq \operatorname{diam}(X) \cdot(1+|\alpha|) e^{3 N \log (|\alpha|)}$. Comme

$$
-\frac{\log \left|t-t^{\prime}\right|}{\log 3}>N
$$

nous avons

$$
\left|f(t)-f\left(t^{\prime}\right)\right| \leq C\left|t-t^{\prime}\right|^{\delta},
$$

où $C=\operatorname{diam}(X) \cdot(1+|\alpha|)$.

Calcul de la dimension de Hausdorff. Comme $\operatorname{Fr}(\mathcal{E})$ est l'union de six régions qui sont chacune l'image de $X$ par une transformation affine (voir lemme 2), nous avons $\operatorname{dim}_{\mathrm{H}}(X)=\operatorname{dim}_{\mathrm{H}}(\operatorname{Fr}(\mathcal{E}))$. L'ensemble $X=\bigcup_{i=0}^{2} f_{i}(X)$ entre dans le cadre des compacts invariants par des similitudes (il est stable par les $f_{i}$ ). La dimension de Hausdorff de cette classe de compact est majorée par sa dimension fractale et dans des cas, elle lui est égale.

ThÉORÈme 2 (voir [2], [9]). Soit $A$ un sous-ensemble de $\mathbb{C}$ tel que $A=$ $\bigcup_{i=0}^{n} h_{i}(A)$ est le compact invariant par des similitudes $h_{i}$ de coefficients de similitudes $r_{i}$ (i.e. $\left.\forall x, y \in \mathbb{C},\left|h_{i}(x)-h_{i}(y)\right|=r_{i}|x-y|\right)$. Alors $\operatorname{dim}_{\mathrm{H}}(A) \leq s$ où $s$ est l'unique réel vérifiant $\sum_{i=0}^{n} r_{i}^{s}=1$. Si de plus il existe un ouvert $O$ de $\mathbb{C}$ tel que $\forall i, h_{i}(O) \subset O$ et $h_{i}(O) \cap h_{j}(O)=\emptyset, \forall i \neq j$, alors $\operatorname{dim}_{\mathrm{H}}(A)=s$.

Soit $\left(a_{i}\right)_{0 \leq i \leq m} \in \mathcal{N}_{\mathrm{f}}$. Notons

$$
C_{a_{0} \ldots a_{m}}=\left\{z=\sum_{i=0}^{m} a_{i} \alpha^{i}+\sum_{i=m+1}^{\infty} d_{i} \alpha^{i} \text { où } a_{0} \ldots a_{m} d_{m+1} d_{m+2} \ldots \in \mathcal{N}\right\} .
$$

Proposition 3. Soit $U=\operatorname{int}\left(C_{0000100000}\right)$ et $O=\bigcup_{p=1}^{\infty} g_{i_{p}} \circ \ldots \circ g_{i_{2}} \circ g_{i_{1}}(U)$ où $i_{1}, \ldots, i_{p}=0,1,2$. Alors

(1) $g_{i}(O) \subset O, i=0,1,2$.

(2) $g_{i}(O) \cap g_{j}(O)=\emptyset$ si $i \neq j$.

Lemme 8. Soit $a_{0} \ldots a_{m} \in \mathcal{N}_{\mathrm{f}}, m \geq 3$. Si $\operatorname{int}\left(C_{a_{0} \ldots a_{m}}\right)=g_{i_{p}} \circ \ldots \circ g_{i_{1}}(U)$, alors $a_{0} \ldots a_{m}$ ne contient pas cinq "0" consécutifs sauf peut être à la fin.

Preuve. Un simple calcul montre que

$$
\begin{array}{ll}
g_{0}\left(C_{000 a_{3} \ldots a_{m}}\right)=C_{000010 a_{3} \ldots a_{m}}, & g_{0}\left(C_{010 a_{3} \ldots a_{m}}\right)=C_{010001 a_{3} \ldots a_{m}}, \\
g_{1}\left(C_{000 a_{3} \ldots a_{m}}\right)=C_{0101010 a_{3} \ldots a_{m}}, & g_{1}\left(C_{010 a_{3} \ldots a_{m}}\right)=C_{0000101 a_{3} \ldots a_{m}}, \\
g_{2}\left(C_{000 a_{3} \ldots a_{m}}\right)=C_{000110 a_{3} \ldots a_{m}}, & g_{2}\left(C_{010 a_{3} \ldots a_{m}}\right)=C_{010101 a_{3} \ldots a_{m}} .
\end{array}
$$

Montrons le lemme 8 par recurrence sur $p$.

Si $p=1$, c'est évident. 
Supposons la propriété du lemme 8 est vraie à l'ordre $p-1$. On a $g_{i_{p}} \circ \ldots \circ$ $g_{i_{1}}(U)=g_{i_{p}}\left(\operatorname{int}\left(C_{d_{0} \ldots d_{l}}\right)\right)$.

- Si $i_{p}=0$, alors $g_{i_{p}} \circ \ldots \circ g_{i_{1}}(U)$ satisfait la propriété du lemme 8 sauf si $d_{0} \ldots d_{l}=0000000 d_{7} \ldots d_{l}$ ou $d_{0} \ldots d_{l}=010110 d_{6} \ldots d_{l}$.

- Si $i_{p}=1$ ou 2 , alors $g_{i_{p}} \circ \ldots \circ g_{i_{1}}(U)$ satisfait la propriété du lemme 8 sauf si $d_{0} \ldots d_{l}=0000000 d_{7} \ldots d_{l}$ ou $d_{0} \ldots d_{l}=010110110 d_{9} \ldots d_{l}$.

Il est facile de voir que dans ces cas $\operatorname{int}\left(C_{d_{0} \ldots d_{l}}\right)$ ne peut pas être sous la forme $g_{i_{p-1}} \circ \ldots \circ g_{i_{1}}(U)$.

Lemme 9. Soient $a_{0} \ldots a_{m}$ et $b_{0} \ldots b_{k} \in \mathcal{N}_{\mathrm{f}}$ où $k, m \geq 3$, et $a_{0} a_{1} a_{2}, b_{0} b_{1} b_{2}$ $\in\{000,010\}$. S'il existe $i_{1}, \ldots, i_{p} \in\{0,1,2\}$ tels que

$$
g_{i_{p}} \circ \ldots \circ g_{i_{1}}\left(\operatorname{int}\left(C_{b_{0} \ldots b_{k}}\right)\right)=\operatorname{int}\left(C_{a_{0} \ldots a_{m}}\right) \text {, }
$$

alors $\left(i_{1}, \ldots, i_{p}\right)$ est uniquement déterminé.

Preuve. D'après la définition des $g_{i}$ (lemme 8), on a :

1. Si $b_{0} b_{1} b_{2}=000$, alors

- si $a_{m-k}=0$ et $a_{m-k-1}=0$ alors $i_{1}=0$,

- si $a_{m-k}=0$ et $a_{m-k-1}=1$ alors $i_{1}=1$,

- si $a_{m-k}=1$ alors $i_{1}=2$.

2. Si $b_{0} b_{1} b_{2}=010$, alors

- si $a_{m-k}=0$ alors $i_{1}=0$,

- si $a_{m-k}=1$ et $\left(a_{m-k-1}, a_{m-k-2}\right)=(0,0)$ alors $i_{1}=1$,

- si $a_{m-k}=1$ et $\left(a_{m-k-1}, a_{m-k-2}\right) \neq(0,0)$ alors $i_{1}=2$.

D'où $i_{1}$ est uniquement déterminé. Nous appliquons le même procédé à $g_{i_{1}}\left(\operatorname{int}\left(C_{b_{0} \ldots b_{k}}\right)\right)$ et $\operatorname{int}\left(C_{a_{0} \ldots a_{m}}\right)$ pour obtenir que $i_{2}$ est uniquement déterminé. En continuant le même procédé, nous obtenons le lemme.

Preuve de la proposition 3. (1) est évident.

(2) Supposons que $g_{i_{p}} \circ \ldots \circ g_{i_{1}}(U)=\operatorname{int}\left(C_{a_{0} \ldots a_{m}}\right)$ et $g_{j_{s}} \circ \ldots \circ g_{j_{1}}(U)=$ $\operatorname{int}\left(C_{d_{0} \ldots d_{k}}\right)$ où $m \geq k$. Supposons que

$$
g_{i_{p}} \circ \ldots \circ g_{i_{1}}(U) \cap g_{j_{s}} \circ \ldots \circ g_{j_{1}}(U) \neq \emptyset .
$$

Comme l'ensemble des nombres complexes qui ont au moins deux $\alpha$-développements est de mesure nulle [16], nous avons $a_{i}=d_{i}$ pour $0 \leq i \leq k$. Puisque $a_{k}=a_{k-1}=$ $\ldots=a_{k-4}=0$ et en vertu du lemme 8 , nous avons $k=m$. Le lemme 9 implique que $\left(i_{p}, \ldots, i_{1}\right)=\left(j_{s}, \ldots, j_{1}\right)$. Ceci termine la preuve.

Nous déduisons de la proposition 3 et du théorème 2 que $\operatorname{dim}_{\mathrm{H}}(X)=s$ où $s$ vérifie

$$
2|\alpha|^{3 s}+|\alpha|^{4 s}=1
$$


Nous en concluons que $\operatorname{dim}_{H}(X)=\log \varrho / \log |\alpha|=1.09336 \ldots$ où $\varrho$ est la racine réelle maximale du polynôme $X^{4}+2 X^{3}-1=0$. Nous retrouvons un résultat de S. Ito et M. Kimura [10].

Remarque. Soit $\gamma$ un nombre de Pisot réel ou complexe (entier algébrique de module $>1$ dont tous les conjugués au sens de Galois, à l'exception de $\bar{\gamma}$, sont de module $<1)$. Soit $A$ un sous-ensemble fini d'entiers algébriques de $\mathbb{Q}(\gamma)$. Soit $\Xi=\left\{\sum_{i=0}^{\infty} a_{i} \gamma^{-i} \mid\left(a_{i}\right) \in A^{\mathbb{N}}\right\}$. Il est connu ([20], [15]) qu'il existe un automate fini $\mathcal{L}$ qui reconnaît toutes les suites $\left(a_{i}, b_{i}\right)_{i \in \mathbb{N}},\left(a_{i}\right),\left(b_{i}\right) \in A^{\mathbb{N}}$ telles que $\sum_{i=0}^{\infty} a_{i} \gamma^{-i}=$ $\sum_{i=0}^{\infty} b_{i} \gamma^{-i}$. Il est intéressant de voir si on peut utiliser le même raisonnement que ci-dessus pour paramétriser la frontière de $\Xi$ et calculer sa dimension de Hausdorff.

4.1. La frontière de $\mathcal{E}$ est un quasi-cercle. Nous commençons par donner quelques définitions (voir [14]).

Dans le plan complexe, on considère le quadrilatère $Q=Q\left(z_{1}, z_{2}, z_{3}, z_{4}\right)$ de sommets $z_{i}$. Soit $f$ une fonction conforme de $Q$ dans un rectangle $R$. Si $f\left(z_{i}\right), i \in$ $\{1,2,3,4\}$, coïncident avec les sommets de $R$, alors on dit que $f$ est canonique et $R$ est appelé rectangle canonique de $Q$.

Il est connu [14] que tout quadrilatère possède une fonction conforme canonique, unique modulo des similitudes.

On suppose que $R=\{x+i y \mid 0<x<a, 0<y<b\}$ est rectangle canonique de $Q\left(z_{1}, z_{2}, z_{3}, z_{4}\right)$ tel que le côté $\left[z_{1}, z_{2}\right]$ correspond au segment $[0, a]$.

On appelle

$$
a / b=M\left(Q\left(z_{1}, z_{2}, z_{3}, z_{4}\right)\right)
$$

le module du quadrilatère $Q\left(z_{1}, z_{2}, z_{3}, z_{4}\right)$.

On montre que le module d'un quadrilatère est indépendant du choix du rectangle canonique associé.

Soit $A$ un domaine. On considère $\mathcal{K}$ l'ensemble de tous les quadrilatères $Q=$ $Q\left(z_{1}, z_{2}, z_{3}, z_{4}\right)$ tels que $\bar{Q} \subset A$ et $f$ un homéomorphisme qui préserve le sens. On pose

$$
M_{f}=\sup _{\mathcal{K}} \frac{M(f(Q))\left(f\left(z_{1}\right), f\left(z_{2}\right), f\left(z_{3}\right), f\left(z_{4}\right)\right)}{M\left(Q\left(z_{1}, z_{2}, z_{3}, z_{4}\right)\right)}
$$

DÉFINITION 1. Un homéomorphisme préservant l'orientation est dit quasi-conforme si $M_{f}$ est fini. En particulier si $M_{f}=1$ alors $f$ est conforme.

DÉfinition 2. Un courbe de Jordan $\mathcal{J}$ est un quasi-cercle si elle est l'image d'un cercle par un homéomorphisme quasi-conforme. Le domaine entouré par un quasi-cercle est appelé quasi-disque. 
DÉfinition 3. Soient $x$ et $y$ deux éléments de $\mathcal{J}$, soit $\mathrm{I}(x, y)$ l'arc de $\mathcal{J}$ orienté positivement et $\operatorname{diam}(\mathrm{I}(x, y))$ le diamètre de cet arc. On dit que $\mathcal{J}$ vérifie les conditions d'Ahlfors s'il existe un réel positif $k$ tel que

$$
\forall x, y \in \mathcal{J}, \quad \min (\operatorname{diam}(\mathrm{I}(x, y)), \operatorname{diam}(\mathrm{I}(y, x))) \leq k|x-y| .
$$

ThÉORÈme 3 ([14]). Si $\mathcal{J}$ vérifie les conditions d'Ahlfors, alors $\mathcal{J}$ est un quasi-cercle.

Nous allons donc montrer que $\operatorname{Fr}(\mathcal{E})$ vérifie les conditions d'Ahlfors; pour cela, nous avons besoin du lemme suivant.

LEMME 10. Il existe un réel strictement positif $k$ tel que si $0 \leq t_{0} \leq t_{1} \leq t_{2} \leq 1$, alors $\left|f\left(t_{1}\right)-f\left(t_{0}\right)\right| \leq k\left|f\left(t_{2}\right)-f\left(t_{0}\right)\right|$.

Preuve. Il est facile de vérifier que

$$
f_{0} \circ f_{2}(z)=f_{0} \circ f_{0}(z)+\alpha^{6}, \quad f_{1} \circ f_{0}(z)=f_{0} \circ f_{1}(z)+\alpha^{6} .
$$

D'où

$$
f[2 / 9,1 / 3]=f_{0} \circ f_{2}(X)=f_{0} \circ f_{0}(X)+\alpha^{6}=f[0,1 / 9]+\alpha^{6},
$$

et

$$
f[1 / 3,1 / 3+1 / 9]=f_{1} \circ f_{0}(X)=f_{0} \circ f_{1}(X)+\alpha^{6}=f[1 / 9,2 / 9]+\alpha^{6} .
$$

Par conséquent,

$$
f[2 / 9,1 / 3+1 / 9]=f[0,2 / 9]+\alpha^{6} .
$$

De même, en utilisant la symétrie de $X$, nous obtenons

$$
f[1-2 / 9,1]=f[1-4 / 9,1-2 / 9]+\alpha^{6} .
$$

Posons [0,1] $=A_{1} \cup A_{2} \cup A_{3} \cup A_{4} \cup A_{5}$ où $A_{1}=[0,1 / 3], A_{2}=[2 / 9,1 / 3+1 / 9]$, $A_{3}=[1 / 3,2 / 3], A_{4}=[1-4 / 9,1-2 / 9]$ et $A_{5}=[2 / 3,1]$. Donc $X=\bigcup_{i=1}^{5} f\left(A_{i}\right)$.

Soient $t_{0}, t_{1}$ et $t_{2}$ trois éléments de $[0,1]$ tels que $0 \leq t_{0} \leq t_{1} \leq t_{2} \leq 1$. Nous avons donc deux cas à étudier :

(1) Il existe $i$ appartenant à $\{1,2,3,4,5\}$ tel que $f\left(t_{0}\right)$ et $f\left(t_{2}\right)$ appartiennent à $f\left(A_{i}\right)$.

(2) Il n'existe pas $i$ tel que $f\left(t_{0}\right)$ et $f\left(t_{2}\right)$ appartiennent à $f\left(A_{i}\right)$.

Supposons que l'on est dans le cas (2). Soit $p$ le minimum des distances entre deux points de $X$ vérifiant (2); d'après la construction des $A_{i}, p$ est strictement positif. Puisque $p \leq\left|f\left(t_{2}\right)-f\left(t_{0}\right)\right|$, nous avons

$$
\left|f\left(t_{1}\right)-f\left(t_{0}\right)\right| \leq \frac{\operatorname{diam}(X)}{p} \cdot\left|f\left(t_{2}\right)-f\left(t_{0}\right)\right| .
$$

Il suffit donc de prendre $k=\operatorname{diam}(X) / p$. 
Si on est dans le cas (1), on peut toujours se ramener au cas où $t_{0}$ et $t_{2}$ appartiennent à $A_{i}$ où $i$ est un élément de $\{1,3,5\}$, car $f\left(A_{2}\right)=f[0,2 / 9]+\alpha^{6}$ et $f\left(A_{4}\right)=f[1-2 / 9,1]-\alpha^{6}$.

Posons $i=2 s+1, s \in\{0,1,2\}$ et définissons l'application $h$ de $[0,1]$ dans lui même par

$$
h(t)=3 t-s \quad \text { si } t \in A_{2 s+1} .
$$

Donc

$$
f_{s} \circ f\left(h\left(t_{0}\right)\right)=f\left(t_{0}\right), \quad f_{s} \circ f\left(h\left(t_{2}\right)\right)=f\left(t_{2}\right) .
$$

De même, nous avons $f_{s} \circ f\left(h\left(t_{1}\right)\right)=f\left(t_{1}\right)$, car $t_{1} \in A_{2 s+1}$. Pour avoir le lemme, il suffit d'avoir

$$
\left|f\left(h\left(t_{1}\right)\right)-f\left(h\left(t_{0}\right)\right)\right| \leq k\left|f\left(h\left(t_{2}\right)\right)-f\left(h\left(t_{0}\right)\right)\right| .
$$

Par ailleurs,

$$
\left|\alpha^{-3}\right| \cdot\left|f\left(t_{0}\right)-f\left(t_{2}\right)\right| \leq\left|f\left(h\left(t_{0}\right)\right)-f\left(h\left(t_{2}\right)\right)\right| \leq\left|\alpha^{-4}\right| \cdot\left|f\left(t_{0}\right)-f\left(t_{2}\right)\right| .
$$

Comme $\alpha$ est de module inférieur strictement à 1 , en appliquant $h$ un nombre fini de fois, on obtient un couple $\left(h^{n}\left(t_{0}\right), h^{n}\left(t_{2}\right)\right)$ appartenant à $X$ et vérifiant

$$
\left|f\left(h^{n}\left(t_{0}\right)\right)-f\left(h^{n}\left(t_{2}\right)\right)\right| \geq p .
$$

On prend $k=\operatorname{diam}(X) / p$ et on obtient

$$
\left|f\left(h^{n}\left(t_{1}\right)\right)-f\left(h^{n}\left(t_{0}\right)\right)\right| \leq k\left|f\left(h^{n}\left(t_{0}\right)\right)-f\left(h^{n}\left(t_{2}\right)\right)\right| .
$$

Comme $t_{1}$ appartient à $A_{i}$, nous avons $\left|f\left(t_{1}\right)-f\left(t_{0}\right)\right| \leq k\left|f\left(t_{2}\right)-f\left(t_{0}\right)\right|$.

Un corollaire immédiat du lemme 10 est le suivant.

CoRollaire 2. Il existe un réel strictement positif $k$ tel que pour tout réel $t, t^{\prime}, t_{0}$ et $t_{2}$ vérifiant $0 \leq t_{0} \leq t \leq t^{\prime} \leq t_{2} \leq 1$, on a $\left|f(t)-f\left(t^{\prime}\right)\right| \leq k\left|f\left(t_{2}\right)-f\left(t_{0}\right)\right|$.

THÉORÈme 4 . Il existe un réel positif $k$ tel que pour tout $x$ et $y$ appartenant à $\operatorname{Fr}(\mathcal{E})$, on a $\min (\operatorname{diam}(\mathrm{I}(x, y)), \operatorname{diam}(\mathrm{I}(y, x))) \leq k|x-y|$.

Preuve. Comme $f_{2}(X)-\alpha$ est inclus dans $\mathcal{E} \cap(\mathcal{E}-\alpha)$, la frontière de $\mathcal{E}$ est l'union de six $\operatorname{arcs} B_{0}=X \cup Y, B_{1}=Y \cup Z, B_{2}=Z \cup\left(f_{2}(X)-\alpha\right), B_{3}=X^{\prime} \cup Y^{\prime}$, $B_{4}=Y^{\prime} \cup Z^{\prime}, B_{5}=Z^{\prime} \cup f_{0}(X)$.

D'après la figure 5 , on remarque que ces arcs sont similaires à $X$ et on montre en utilisant le lemme 2 qu'il existe des applications affines $\mathcal{H}_{i}$ de $\mathbb{C}$ dans $\mathbb{C}, i$ élément de $\{0,1,2,3,4,5\}$, telles que l'image de $B_{i}$ par $\mathcal{H}_{i}$ soit incluse dans $X$. Ces applications sont définies par

$$
\mathcal{H}_{0}(z)=\alpha^{4}+\alpha^{3} z, \quad \mathcal{H}_{1}(z)=\alpha+\alpha^{3}+\alpha^{2} z, \quad \mathcal{H}_{2}(z)=\alpha z,
$$

et

$$
\mathcal{H}_{3}=\mathcal{H}_{0} \circ s, \quad \mathcal{H}_{4}=\mathcal{H}_{1} \circ s, \quad \mathcal{H}_{5}=\mathcal{H}_{2} \circ s,
$$

où $s$ est la symétrie centrale définie sur $\mathcal{E}$. 
En effet, nous avons

$$
\begin{aligned}
\mathcal{H}_{0}(X \cup Y) & =\mathcal{H}_{0}(X \cup(1+\alpha+\alpha X)) \\
& =\left(\alpha^{4}+\alpha^{3} X\right) \cup\left(\alpha+\alpha^{3}+\alpha^{5}+\alpha^{4} X\right) \\
& =f_{0}(X) \cup f_{1}(X) \subset X, \\
\mathcal{H}_{1}(Y \cup Z) & =\mathcal{H}_{1}\left((1+\alpha+\alpha X) \cup\left(\alpha^{3}+\alpha^{2} X\right)\right) \\
& =\left(\alpha^{3}+\alpha^{4}+\alpha^{3} X\right) \cup\left(\alpha+\alpha^{3}+\alpha^{5}+\alpha^{4} X\right) \\
& =f_{2}(X) \cup f_{1}(X) \subset X, \\
\mathcal{H}_{2}\left(Z \cup\left(f_{2}(X)-\alpha\right)\right) & =\mathcal{H}_{2}\left(\left(\alpha^{3}+\alpha^{2} X\right) \cup\left(1+\alpha^{2}+\alpha^{4}+\alpha^{3} X\right)\right) \\
& =\left(\alpha^{4}+\alpha^{3} X\right) \cup\left(\alpha+\alpha^{3}+\alpha^{5}+\alpha^{4} X\right) \\
& =f_{0}(X) \cup f_{1}(X) \subset X .
\end{aligned}
$$

Nous avons

$$
\mathcal{H}_{3}\left(X^{\prime} \cup Y^{\prime}\right)=\mathcal{H}_{0}\left(s\left(X^{\prime} \cup Y^{\prime}\right)\right)=\mathcal{H}_{0}(X \cup Y)
$$

De même $\mathcal{H}_{4}\left(Y^{\prime} \cup Z^{\prime}\right)=\mathcal{H}_{1}(Y \cup Z)$ et $\mathcal{H}_{5}\left(Z^{\prime} \cup f_{0}(X)\right)=\mathcal{H}_{2}\left(f_{2}(X)-\alpha\right)$.

Soient $x$ et $y$ deux éléments de $\operatorname{Fr}(\mathcal{E})$. Alors nous avons deux cas :

(1) $x$ et $y$ appartiennent au même arc $B_{i}$. Dans ce cas, $\mathcal{H}_{i}(x)$ et $\mathcal{H}_{i}(y)$ appartiennent à $X$ et il résulte du corollaire 2 que

$$
\min \left(\operatorname{diam}\left(\mathrm{I}\left(\mathcal{H}_{i}(x), \mathcal{H}_{i}(y)\right)\right), \operatorname{diam}\left(\mathrm{I}\left(\mathcal{H}_{i}(y), \mathcal{H}_{i}(x)\right)\right)\right) \leq k\left|\mathcal{H}_{i}(x)-\mathcal{H}_{i}(y)\right|
$$

d'où le résultat.

(2) $x$ et $y$ n'appartiennent pas au même arc $B_{i}$. Donc, d'après la construction des $B_{i}, x$ et $y$ n'appartiennent pas à deux arcs successifs de $\operatorname{Fr}(\mathcal{E})$ (i.e. $(x, y) \notin$ $\left.(X \times Y) \cup(Y \times Z) \cup\left(Z \times X^{\prime}\right) \cup\left(X^{\prime} \times Y^{\prime}\right) \cup\left(Y^{\prime} \times Z^{\prime}\right) \cup\left(Z^{\prime} \times X\right)\right)$. D'où il existe un réel strictement positif $d$ tel que $|x-y| \geq d$. Il suffit donc de prendre $k=\operatorname{diam}(\operatorname{Fr}(\mathcal{E})) / d$ pour avoir le théorème.

Il est connu [14] que la classe des domaines qui sont des quasi-disques coïncide avec celle des domaines uniformes. Il s'en suit que $\operatorname{int}(\mathcal{E})$ est un domaine uniforme, c'est-à-dire, il existe deux réels positifs $a$ et $b$ tels que tout $z_{1}$ et $z_{2}$ appartenant à $\operatorname{int}(\mathcal{E})$ peuvent être joints par un $\operatorname{arc} \eta$ dans $\operatorname{int}(\mathcal{E})$ qui vérifie les deux propriétés suivantes :

- La longueur euclidienne $l(\eta)$ de $\eta$ satisfait l'inégalité $l(\eta) \leq a\left|z_{1}-z_{2}\right|$.

- $\forall z \in \eta, \min \left(l\left(\eta_{1}\right), l\left(\eta_{2}\right)\right) \leq b \cdot d(z, \operatorname{Fr}(\mathcal{E}))$ où $\eta_{1}$ et $\eta_{2}$ sont les deux arcs de $\eta \backslash z$.

Remarque. Il a été prouvé dans [3] que la frontière du fractal du dragon est un quasi-cercle. Ceci motive la question suivante : Parmi les courbes générées par la méthode de Dekking, quelles sont celles qui sont quasi-cercles? 
5. Les points strictement extrémaux du fractal de Rauzy. Dans cette section nous construisons les points strictement extrémaux du fractal de Rauzy, ce qui permet de trouver l'enveloppe convexe du fractal de Rauzy et se généralise aux $n$-fractals du dragon, $n \geq 1$.

Dans le plan complexe, nous considérons une droite $\Delta_{a}$ passant par l'origine, de vecteur directeur $\vec{u}$ et de direction un réel $a \in\left[0,2 \pi\left[\right.\right.$. Soit $p_{a}$ la projection orthogonale de $\mathcal{E}$ sur $\Delta_{a}: \forall z \in \mathcal{E}, p_{a}(z)=c_{a}(z) \vec{u}$, où $c_{a}(z) \in \mathbb{R}$. Comme $\mathcal{E}$ est compact, le maximum des $c_{a}(z), z \in \mathcal{E}$, est atteint en au moins un point $x$ appartenant à $\mathcal{E}$.

DÉfinition 4. Sous les mêmes hypothèses, un point $x \in \mathcal{E}$ est dit strictement extrémal pour la direction $a$ si $c_{a}(x)=\max \left\{c_{a}(z) ; z \in \mathcal{E}\right\}$.

Remarque. Un point strictement extrémal de $\mathcal{E}$ appartient à $\operatorname{Fr}(\mathcal{E})$.

Construction d'un point strictement extrémal. Soit $\alpha=|\alpha| e^{i \phi}$ où $\phi$ est un argument de $\alpha$ appartenant à l'ensemble $[0,2 \pi[(\phi / \pi \sim 0.69)$. Soit $a \in[0,2 \pi[$ et $z \in \mathcal{E}$ tel que $z=\sum_{n=3}^{\infty} \varepsilon_{n} \alpha^{n}$. Alors

$$
p_{a}(z)=\operatorname{Re}\left(z e^{-i a}\right) \vec{u}=\left(\sum_{n=3}^{\infty} \varepsilon_{n}|\alpha|^{n} \cos (n \phi-a)\right) \vec{u} .
$$

Soit $\left(a_{n}\right)_{n \geq 3}$ une suite dont les termes sont dans $\{0,1\}$ et vérifient

$$
\left\{\begin{array}{l}
a_{n}=1 \quad \text { si } \cos (n \phi-a)>0, \\
a_{n}=0 \text { si } \cos (n \phi-a)<0, \\
a_{n} \text { arbitraire dans }\{0,1\} \quad \text { si } \cos (n \phi-a)=0 .
\end{array}\right.
$$

Proposition 4. Soit $\left(a_{n}\right)_{n \geq 3}$ une des suites définies ci-dessus. Alors $\left(a_{n}\right)_{n \geq 3} \in$ $\mathcal{N}$, et $\sum_{n=3}^{\infty} a_{n} \alpha^{n}$ est un point strictement extrémal pour la direction a.

Preuve. Montrons que $\left(a_{n}\right)_{n \geq 3} \in \mathcal{N}$. Supposons qu'il existe $n \geq 3$ tel que $a_{n}=a_{n+1}=1$; d'où $\cos (n \phi-a)$ et $\cos ((n+1) \phi-a)$ sont positifs. Par conséquent il existe deux entiers relatifs $k$ et $k^{\prime}$ tels que

$$
-\frac{\pi}{2}+2 k \pi \leq n \phi-a \leq \frac{\pi}{2}+2 k \pi \quad \text { et } \quad-\frac{\pi}{2}+2 k^{\prime} \pi \leq(n+1) \phi-a \leq \frac{\pi}{2}+2 k^{\prime} \pi,
$$

ou encore

$$
2 k \leq n \frac{\phi}{\pi}-\frac{a}{\pi}+\frac{1}{2} \leq 2 k+1 \quad \text { et } \quad 2 k^{\prime} \leq(n+1) \frac{\phi}{\pi}-\frac{a}{\pi}+\frac{1}{2} \leq 2 k^{\prime}+1 .
$$

Nous avons $k^{\prime} \geq k \operatorname{car}(n+1) \frac{\phi}{\pi}-\frac{a}{\pi}+\frac{1}{2}>n \frac{\phi}{\pi}-\frac{a}{\pi}+\frac{1}{2}$, d'où

$$
2 k^{\prime}-2 k-1 \leq\left((n+1) \frac{\phi}{\pi}-\frac{a}{\pi}+\frac{1}{2}\right)-\left(n \frac{\phi}{\pi}-\frac{a}{\pi}+\frac{1}{2}\right)=\frac{\phi}{\pi}<1,
$$

ce qui implique que $k^{\prime}=k$. 
Par ailleurs

$$
n \frac{\phi}{\pi}-\frac{a}{\pi}+\frac{1}{2}<\left(n \frac{\phi}{\pi}-\frac{a}{\pi}+\frac{1}{2}\right)+\left(2 \frac{\phi}{\pi}-1\right)<(n+1) \frac{\phi}{\pi}-\frac{a}{\pi}+\frac{1}{2}
$$

$\operatorname{car} \phi / \pi \sim 0.69$. Donc $2 k<(n+2) \frac{\phi}{\pi}-\frac{a}{\pi}+\frac{1}{2}-1<2 k+1$, c'est-à-dire $2 k+1<$ $(n+2) \frac{\phi}{\pi}-\frac{a}{\pi}+\frac{1}{2}<2 k+2$. Il en résulte que $\cos ((n+2) \phi-a)<0$, d'où $a_{n+2}=0$, ce qui implique que $\left(a_{n}\right)_{n \geq 3} \in \mathcal{N}$.

Par ailleurs, soit $\left(\varepsilon_{n}\right)_{n \geq 3}$ dans $\mathcal{N}$. De la relation (1) et de la définition de $\left(a_{n}\right)$, nous déduisons que

$$
\sum_{n=3}^{\infty} \varepsilon_{n}|\alpha|^{n} \cos (n \phi-a) \leq \sum_{n=3}^{\infty} a_{n}|\alpha|^{n} \cos (n \phi-a)
$$

Par conséquent $\sum_{n=3}^{\infty} a_{n} \alpha^{n}$ est un point strictement extrémal pour la direction $a$.

REMARque. La preuve montre aussi que la suite $\left(a_{n}\right)_{n \geq 3}$ ne peut pas contenir trois " 0 " consécutifs. Il suffit de remplacer partout $2 k$ (resp. $2 k^{\prime}$ ) par $2 k+1$ (resp. $\left.2 k^{\prime}+1\right)$.

La définition de la suite $\left(a_{n}\right)_{n \geq 3}$ montre qu'a priori, nous pouvons avoir une infinité de points strictement extrémaux pour une direction $a$. Cela est faux. Nous allons prouver que pour une direction donnée, on ne peut avoir plus de deux points strictement extrémaux.

LEMME 11. $\phi / \pi$ est irrationnel.

Preuve. Supposons que $\phi / \pi$ est rationnel, il existe donc un entier naturel $n$ tel que $\alpha^{n}$ soit réel. D'où $\alpha^{n}=\overline{\alpha^{n}}$. Il en résulte que le seul possible conjugué au sens de Galois de $\alpha^{n}$, différent de $\alpha^{n}$ est $\beta^{n}$ où $\beta$ est le conjugué réel de $\alpha$. D'où le corps $\mathbb{Q}\left(\alpha^{n}\right)$ est strictement inclus dans le corps cubique $\mathbb{Q}(\alpha)$. Cela implique que $\mathbb{Q}\left(\alpha^{n}\right)=\mathbb{Q}$ car un corps cubique ne peut pas contenir un corps quadratique. Par conséquent, il existe deux entiers relatifs $m$ et $r$ tels que $m \alpha^{n}-r=0$, d'où le polynôme $Q(X)=m X^{n}-r$ est un multiple du polynôme minimal de $\alpha$. Cela est impossible, car toutes les racines de $Q(X)$ ont le même module.

REMARQUE. Ce lemme est vrai pour $\arg (\gamma) / \pi$ où $\gamma$ est un complexe algébrique de degré 3 ayant au moins un conjugué de module différent de celui de $\gamma$.

Corollaire 3. Soit $a \in[0,2 \pi[$. Alors l'ensemble $\{n \geq 3 \mid \cos (n \phi-a)$ $=0\}$ est soit vide, soit réduit à un élément.

Preuve. Supposons qu'il existe deux entiers différents $m$ et $n$ supérieurs ou égaux à 3 tels que $\cos (n \phi-a)=\cos (m \phi-a)=0$. Par conséquent, il existe $s \in \mathbb{Z}$ tel que $\phi / \pi=s /(n-m) \in \mathbb{Q}$, ce qui contredit le lemme 11 . 
Maintenant, nous sommes en mesure d'expliciter les points strictement extrémaux.

Premier CAS. Si $\{n \geq 3 \mid \cos (n \phi-a)=0\}=\emptyset$, alors nous avons un seul point strictement extrémal pour la direction $a$. Ce point est $x_{a}=\sum_{n=3}^{\infty} a_{n} \alpha^{n}$, où

$$
a_{n}= \begin{cases}1 & \text { si } \cos (n \phi-a) \geq 0 \\ 0 & \text { sinon }\end{cases}
$$

ou encore

$$
a_{n}= \begin{cases}1 & \text { si } \exists k \in \mathbb{Z}, 2 k-1 \leq n \frac{\phi}{\pi}-\frac{a}{\pi}-\frac{1}{2} \leq 2 k, \\ 0 & \text { sinon. }\end{cases}
$$

Comme pour tout entier $n \geq 3$ et $k \in \mathbb{Z}, n \frac{\phi}{\pi}-\frac{a}{\pi}-\frac{1}{2} \notin\{2 k-1,2 k\} \operatorname{car} \cos (n \phi-a) \neq$ 0 , nous avons

$$
a_{n}=\left(\left[n \frac{\phi}{\pi}-\frac{a}{\pi}-\frac{1}{2}\right]\right) \bmod 2 .
$$

DeuxiÈme CAs. Il existe un unique entier $m \geq 3$ tel que $\cos (m \phi-a)=0$, ou encore $a=m \phi+(1 / 2-p) \pi, p \in \mathbb{Z}$. Dans ce cas, nous obtenons deux points strictement extrémaux qui sont $x_{a}=\sum_{n=3}^{\infty} a_{n} \alpha^{n}$ et $y_{a}=\sum_{n=3}^{\infty} b_{n} \alpha^{n}$ où

$$
a_{n}= \begin{cases}1 & \text { si } \cos (n \phi-a) \geq 0 \\ 0 & \text { sinon }\end{cases}
$$

et

$$
b_{n}= \begin{cases}1 & \text { si } \cos (n \phi-a)>0 \\ 0 & \text { sinon }\end{cases}
$$

Pour tout $n \geq 3$, si $n \neq m$ alors $a_{n}=b_{n}$ et $a_{m}=1, b_{m}=0$. Par conséquent

$$
y_{a}=\sum_{n=3, n \neq m}^{\infty} a_{n} \alpha^{n}
$$

et

$$
x_{a}=\sum_{n=3, n \neq m}^{\infty} a_{n} \alpha^{n}+\alpha^{m}=\sum_{n=3, n \neq m}^{\infty} a_{n} \alpha^{n}+\left|\alpha^{m}\right| e^{i(a-(1 / 2-p) \pi)} .
$$

D'où $x_{a}=y_{a}+\left|\alpha^{m}\right| e^{i(a-\pi / 2)}$ si $p$ est pair, $x_{a}=y_{a}-\left|\alpha^{m}\right| e^{i(a-\pi / 2)}$ si $p$ est impair et $y_{a}=\sum_{n=3, n \neq m}^{\infty} a_{n} \alpha^{n}$ où $a_{n}=\left(\left[n \frac{\phi}{\pi}-\frac{a}{\pi}-\frac{1}{2}\right]\right) \bmod 2$, pour tout $n \geq 3$.

Proposition 5. Soit $x=\sum_{n=3}^{\infty} a_{n} \alpha^{n}$ un point strictement extrémal de $\mathcal{E}$ pour une direction $a \in\left[0,2 \pi\left[\right.\right.$. Alors la suite $\left(a_{n}\right)_{n \geq 3}$ est le codage de $x_{0}=-\frac{a}{2 \pi}+\frac{1}{4}+$ $\frac{3 \phi}{\pi}[1]$ pour la rotation d'angle $\phi /(2 \pi)$ sous la partition $([0,1 / 2[,[1 / 2,1[)$ ou bien (]0,1/2], ]1/2,1]). 
Pr e u ve. Supposons qu'il existe $m \geq 3$ tel que $a=m \phi+(1 / 2-p) \pi, p \in \mathbb{Z}$. Les deux suites correspondant aux deux points strictement extrémaux sont $\left(a_{n}\right)_{n \geq 3}$ et $\left(b_{n}\right)_{n \geq 3}$ où

$$
a_{n}=1 \Leftrightarrow \exists k \in \mathbb{Z},-\pi / 2+2 k \pi \leq n \phi-a \leq \pi / 2+2 k \pi
$$

et

$$
b_{n}=1 \Leftrightarrow \exists k^{\prime} \in \mathbb{Z},-\pi / 2+2 k \pi<n \phi-a<\pi / 2+2 k \pi .
$$

Nous avons $m \phi-a=-\pi / 2+p \pi$.

Si $p$ est pair, alors en vertu du corollaire 3 , nous avons

$$
\forall n \geq 3, \quad a_{n}=1 \Leftrightarrow \exists k \in \mathbb{Z},-\pi / 2+2 k \pi \leq n \phi-a<\pi / 2+2 k \pi
$$

ou encore

$$
\forall n \geq 3, \quad a_{n}= \begin{cases}1 & \text { si } \frac{n \phi}{2 \pi}-\frac{a}{2 \pi}+\frac{1}{4}[1] \in[0,1 / 2[ \\ 0 & \text { sinon. }\end{cases}
$$

D'où la suite $\left(a_{n}\right)_{n \geq 3}=T^{3}\left(\left(c_{n}\right)_{n \in \mathbb{N}}\right)$ (T est l'application de $\{0,1\}^{\mathbb{N}}$ dans lui même qui à une suite $\left(a_{n}\right)$ associe la suite $\left(a_{n+1}\right)$; et $\left(c_{n}\right)$ est le codage de $-\frac{a}{2 \pi}+\frac{1}{4}[1]$ pour la rotation d'angle $\phi /(2 \pi)$ sous la partition ([0,1/2[, [1/2,1[), ou encore $\left(a_{n}\right)_{n \geq 3}$ est le codage de $x_{0}=-\frac{a}{2 \pi}+\frac{1}{4}+\frac{3 \phi}{\pi}$ pour la rotation d'angle $\phi /(2 \pi)$ sous la partition $([0,1 / 2[,[1 / 2,1[)$.

De même la suite $\left(b_{n}\right)_{n \geq 3}$ est le codage de $-\frac{a}{2 \pi}+\frac{1}{4}+\frac{3 \phi}{\pi}[1]$ pour la rotation d'angle $\phi /(2 \pi)$ sous la partition (] $0,1 / 2],] 1 / 2,1])$.

Si $p$ est impair, alors $m \phi-a=\pi / 2+(p-1) \pi$. Dans ce cas, nous avons

$$
a_{n}= \begin{cases}1 & \text { si } \left.\left.\frac{n \phi}{2 \pi}-\frac{a}{2 \pi}+\frac{1}{4}[1] \in\right] 0,1 / 2\right] \\ 0 & \text { sinon }\end{cases}
$$

et

$$
b_{n}= \begin{cases}1 & \text { si } \frac{n \phi}{2 \pi}-\frac{a}{2 \pi}+\frac{1}{4}[1] \in[0,1 / 2[ \\ 0 & \text { sinon }\end{cases}
$$

Dans le cas où $\cos (n \phi-a) \neq 0$ pour tout $n \geq 3$, les deux codages sont les mêmes et coincident avec la suite $\left(a_{n}\right)_{n \geq 3}$ qui est définie par : $a_{n}=\left(\left[n \frac{\Phi}{\pi}-\frac{a}{\pi}-\frac{1}{2}\right]\right) \bmod 2$ pour tout $n \geq 3$.

REMARQUe. Les suites codages d'une rotation d'angle fixe sous la partition $([0,1 / 2[,[1 / 2,1[)$ ou bien (] $0,1 / 2]] 1 / 2,1]$,$) ont plusieurs propriétés (voir [18]), en$ particulier quand l'angle de rotation est irrationnel; ce qui est le cas ici.

6. L'enveloppe convexe fermée de $\mathcal{E}$. Étant donné un ensemble $A \subset \mathbb{C}$, nous appelons enveloppe convexe fermée de $A$ le plus petit convexe fermé contenant $A$; nous le notons par $O(A)$. 
Il y a deux façons de construire l'enveloppe convexe fermée d'un ensemble. La première est avec les barycentres, elle consiste à joindre deux points quelconques de $A$ par un segment.

ThÉORÈme 5. L'ensemble $O(A)$ est la fermeture topologique de l'ensemble

$$
\{t x+(1-t) y \mid t \in[0,1], x, y \in A\} .
$$

La seconde méthode consiste à utiliser les droites d'appui des éléments de la frontière de $A$.

DÉfinition 5. Soient $A$ et $B$ deux parties de $\mathbb{C}$ et $D$ une droite. On dit que $D$ sépare (resp. strictement) $A$ et $B$ si $A$ est dans l'un et $B$ dans l'autre des demi-espaces (resp. ouverts) déterminés par la droite $D$.

DÉfinition 6. Soit $A$ une partie quelconque de $\mathbb{C}$, on appelle droite d'appui de $A$ toute droite $D$ contenant un point $x \in A$ et séparant $\{x\}$ et $A$. Le point $x$ est appelé point d'appui.

Remarque. Une droite d'appui n'existe pas toujours en un point $x \in A$ et si elle existe, elle peut ne pas être unique et avoir plus d'un point d'appui. Un point d'appui appartient à $\operatorname{Fr}(A)$.

ThÉORÈme 6. L'ensemble $O(A)$ est l'intersection des demi-espaces fermés contenant $A$ et déterminés par toutes les droites d'appui des éléments de $A$.

Pour la preuve, nous allons utiliser la proposition suivante (voir [4], p. 35).

Proposition 6. Si $A$ et $B$ sont deux convexes de $\mathbb{C}$ avec $A$ fermé non vide, $B$ compact et $A \cap B=\emptyset$, alors il existe une droite les séparant strictement.

Preuve (du théorème 6 ). Soit $X(A)$ l'intersection des demi-espaces fermés contenant $A$ et déterminés par toutes les droites d'appui des éléments de $A$. Alors $X(A)$ est un convexe fermé contenant $A$. Soit $z \in X(A)-O(A)$. En vertu de la proposition précédente, il existe une droite $D$ qui sépare strictement $\{z\}$ et $O(A)$. Cela implique l'existence d'une droite d'appui $\Delta$ qui sépare $z$ et $A$ strictement ( $\Delta$ est parallèle à $D)$. D'où une contradiction avec la définition de $X(A)$.

Construction de l'enveloppe convexe de $\mathcal{E}$

Proposition 7. L'ensemble des points strictement extrémaux du fractal de Rauzy est égal à l'ensemble de ses points d'appui.

Preuve. Soit $z$ un point d'appui et $D_{z}$ sa droite d'appui. Considérons $(\Delta)$ la droite passant par l'origine et perpendiculaire à $D_{z}$. Soit $a \in[0,2 \pi[$ la direction de $(\Delta)$. Alors $z$ est un point strictement extrémal pour la direction $a$, car sinon, il existe $z^{\prime} \in \mathcal{E}, z^{\prime} \neq z$, tel que $z^{\prime}$ appartient au demi-plan fermé déterminé par $D_{z}$ et ne contenant pas 0 , ce qui est absurde, car $D_{z}$ sépare $\{z\}$ et $\mathcal{E}$. De même si $z$ est un point strictement extrémal pour une direction $a$, alors c'est un point d'appui : sa droite d'appui est la perpendiculaire à la droite de direction $a$. 
Remarque. Comme les points strictement extrémaux de $\mathcal{E}$ sont associés à des suites qui sont codages d'une rotation d'angle irrationnel, pour deux directions différentes, on ne peut pas obtenir le même point strictement extrémal. Il en résulte que chaque point d'appui de $\mathcal{E}$ possède une et une seule droite d'appui. Une droite d'appui possède au plus deux points d'appui.

ThÉORÈme 7. La frontière de $O(\mathcal{E})$ admet un nombre dénombrable de côtés. Chaque côté a pour extrémité les deux points strictment extrémaux pour la même direction $a=m \phi+(1 / 2-p) \pi$ où $m$ est un entier naturel supérieur ou égal à 3 et $p$ un entier relatif.

7. Application au fractal du dragon. Cette méthode peut être appliquée au fractal du dragon $\mathcal{D}_{1}=\left\{\sum_{n=1}^{\infty} a_{n}(-1+i)^{-n} \mid \forall n \geq 1, a_{n} \in\{0,1\}\right\}$. Posons

$$
\psi=\arg \left(\frac{1}{-1+i}\right)=\frac{5 \pi}{4}
$$

Comme ci-dessus, $x=\sum_{n=1}^{\infty} a_{n}(-1+i)^{-n}$ est un point strictement extrémal pour la direction $a \in\left[0,2 \pi\left[\right.\right.$ si et seulement si la suite $\left(a_{n}\right)_{n \geq 1}$ est définie par

$$
a_{n}= \begin{cases}1 & \text { si } \cos (5 n \pi / 4-a)>0 \\ 0 & \text { si } \cos (5 n \pi / 4-a)<0 \\ \text { arbitraire } & \text { si } \cos (5 n \pi / 4-a)=0\end{cases}
$$

Supposons que pour tout $n \geq 1, \cos (5 n \pi / 4-a) \neq 0$. Nous obtenons un unique point strictement extrémal pour la direction $a$, soit

$$
\sum_{n=1}^{\infty} a_{n}(-1+i)^{-n} \quad \text { où } \quad a_{n}= \begin{cases}1 & \text { si } \frac{5 n}{8}-\frac{a}{2 \pi}+\frac{1}{4}[1] \in[0,1 / 2[, \\ 0 & \text { sinon. }\end{cases}
$$

La suite $\left(a_{n}\right)_{n \geq 1}$ est donc périodique de période 8 . C'est le codage de $-\frac{a}{2 \pi}+\frac{1}{4}+\frac{\psi}{\pi}[1]$ pour la rotation d'angle $5 / 8$ pour la partition ([0,1/2[, $[1 / 2,1[)$.

Quand $a$ parcourt l'ensemble $\left[0,2 \pi\left[-\left\{5 n \pi / 4+(1 / 2-p) \pi \mid p \in \mathbb{Z}, n \in \mathbb{N}^{*}\right\}\right.\right.$, l'ensemble des suites $\left(a_{n}\right)_{n \geq 1}$ associées aux points strictement extrémaux parcourt un ensemble fini à 8 éléments que l'on peut calculer explicitement.

Supposons maintenant que $a=5 m \pi / 4+(1 / 2-p) \pi$ où $p \in \mathbb{Z}$ et $m \in \mathbb{N}^{*}$, c'est-à-dire

$$
a \in\{0, \pi / 4, \pi / 2,3 \pi / 4, \pi, 5 \pi / 4,3 \pi / 2,7 \pi / 4\} .
$$


Nous avons $\cos (5 m \pi / 4-a)=0$. Or l'ensemble $\left\{n \in \mathbb{N}^{*} \mid \cos (5 n \pi / 4-a)=0\right\}=$ $\{m+4 k \mid k \in \mathbb{Z}\} \cap \mathbb{N}^{*}$ est infini. Nous avons donc un nombre infini de points strictement extrémaux pour la direction $a$.

Soit $\sum_{n=1}^{\infty} a_{n}(-1+i)^{-n}$ un point strictement extrémal pour $a$. Pour tout $k \in \mathbb{N}^{*}-\{m\}$, nous avons $a_{k+4}+a_{k}=1$. Par conséquent, pour connaître la suite $\left(a_{n}\right)_{n \geq 1}$, il suffit connaître ses quatre premiers termes.

Premier CAS: $a=0$. Dans ce cas, $m=2$ et $p=3$, d'où pour tout $k \in \mathbb{N}, a_{2+4 k}$ est un élément arbitraire de $\{0,1\}$. D'autre part, en utilisant la relation

$$
a_{n}=([5 n / 4-a / \pi-1 / 2]) \bmod 2 \quad \text { si } n \neq m+4 k
$$

nous obtenons $a_{1}=0, a_{3}=1$ et $a_{4}=0$. Il en résulte que les suites correspondant aux points strictement extrémaux pour la direction 0 sont de la forme $\left(a_{n}\right)_{n \geq 1}$ où $a_{1}=a_{4}=a_{7}=0, a_{3}=a_{5}=a_{8}=1$ et $a_{2+4 k}$ est arbitraire pour tout $k \in \mathbb{N}$, et $a_{n+8}=a_{n}$ pour tout $n \neq 2+4 k$.

Nous notons une suite $a_{1} a_{2} \ldots$ de cette forme par $(\overline{0.101 .01})$ (le "." désigne que l'on a un élément arbitraire de $\{0,1\})$.

Deuxième CAS: $a=\pi / 4$. Dans ce cas $m=3$ et $p=4$, d'où pour tout $k \in \mathbb{N}$, $a_{3+4 k}$ est un élément arbitraire de $\{0,1\}$. En vertu de la relation (1), on trouve $a_{1}=0, a_{2}=1$ et $a_{4}=0$. Donc les suites $\left(a_{n}\right)_{n \geq 1}$ sont de la forme $(\overline{01.010 .1})$.

Nous remarquons que $c=\sum_{n=1}^{\infty} a_{n}(-1+i)^{-n}$ où $\left(a_{n}\right)_{n \geq 1}$ est la suite périodique (01101001), est l'unique point strictement extrémal à la fois pour 0 et $\pi / 4$.

TroisiÈme CAS: $a=\pi / 2$. Dans ce cas $m=4$ et $p=5$, d'où $a_{4 k}$ est arbitraire pour tout $k \in \mathbb{N}^{*}$. On trouve $a_{1}=0, a_{2}=1$ et $a_{3}=0$. Par conséquent, les suites $\left(a_{n}\right)_{n \geq 1}$ sont de la forme $(\overline{010.101}$. $)$.

Soit $d=\sum_{n=1}^{\infty} a_{n}(-1+i)^{-n}$ où $\left(a_{n}\right)_{n \geq 1}$ est la suite périodique $(\overline{01001011})$. Alors $d$ est l'unique point strictement extrémal à la fois pour $\pi / 4$ et $\pi / 2$.

QuATRIÈme CAS: $a=3 \pi / 4$. Dans ce cas nous avons $m=1$ et $p=1$, d'où pour tout $k \in \mathbb{N}, a_{1+4 k}$ est un élément arbitraire de $\{0,1\}$ et pour tout $n \neq 1+4 k$, $a_{n}=([5 n / 4-5 / 4]) \bmod 2$. D'où $a_{2}=1, a_{3}=0$ et $a_{4}=1$. Il en résulte que les suites $\left(a_{n}\right)_{n \geq 1}$ sont de la forme $(\overline{.101 .010})$.

La point $e=\sum_{n=1}^{\infty} a_{n}(-1+i)^{-n}$ où $\left(a_{n}\right)_{n \geq 1}$ est la suite périodique $(\overline{01011010})$ est l'unique point strictement extrémal à la fois pour $\pi / 2$ et $3 \pi / 4$.

D'autre part, en vertu de $(*)$, un point $\sum_{n=1}^{\infty} a_{n}(-1+i)^{-n}$ est strictement extrémal pour la direction $a$ si et seulement si $\sum_{n=1}^{\infty}\left(1-a_{n}\right)(-1+i)^{-n}$ est strictement extrémal pour la direction $\pi-a$. Il en découle que les points strictement extrémaux respectivement pour les directions $\pi, 5 \pi / 4,3 \pi / 2,7 \pi / 4$ se déduisent de 
ceux associés respectivement aux directions $0, \pi / 4, \pi / 2,3 \pi / 4$. Les points $c^{\prime}=\sum_{n=1}^{\infty} a_{n}(-1+i)^{-n}, \quad d^{\prime}=\sum_{n=1}^{\infty} b_{n}(-1+i)^{-n}$ et
$\sum_{n=1}^{\infty} c_{n}(-1+i)^{-n}$ où

$$
\left(a_{n}\right)=(\overline{10010110}), \quad\left(b_{n}\right)=(\overline{10110100}), \quad\left(c_{n}\right)=(\overline{10100101})
$$

sont respectivement les seuls points strictement extrémaux associés respectivement à la fois à $\pi$ et $5 \pi / 4,5 \pi / 4$ et $3 \pi / 2,3 \pi / 2$ et $7 \pi / 4$.

De même, nous montrons que $g=\sum_{n=1}^{\infty} d_{n}(-1+i)^{-n}$ où $\left(d_{n}\right)_{n \geq 1}=(\overline{11010010})$ et $g^{\prime}=\sum_{n=1}^{\infty}\left(1-d_{n}\right)(-1+i)^{-n}$ sont respectivement les seuls points strictement extrémaux à la fois pour $3 \pi / 4$ et $\pi, 7 \pi / 4$ et 0 .

Par conséquent, nous avons la proposition suivante.

Proposition 8. L'enveloppe convexe fermée du fractal du dragon est un polygone convexe; c'est exactement l'octogone dont les 8 côtés ont pour extrémités les points $c, d, e, g, c^{\prime}, d^{\prime}, e^{\prime}, g^{\prime}$.

Nous pouvons facilement calculer les nombres complexes $c, d, e, g, c^{\prime}, d^{\prime}$, $e^{\prime}, g^{\prime}$, car ils ont chacun un développement périodique en base $-1+i$. Posons $-1+i=\gamma$; alors

$$
\begin{aligned}
& c=\frac{\gamma^{2}+\gamma^{3}+\gamma^{5}+\gamma^{8}}{1-\gamma^{8}}=\frac{7+6 i}{15}, \quad d=\frac{\gamma^{2}+\gamma^{5}+\gamma^{7}+\gamma^{8}}{1-\gamma^{8}}=\frac{2+11 i}{15}, \\
& e=\frac{\gamma^{2}+\gamma^{4}+\gamma^{5}+\gamma^{7}}{1-\gamma^{8}}=\frac{-3+11 i}{15}, \quad g=\frac{\gamma+\gamma^{2}+\gamma^{4}+\gamma^{7}}{1-\gamma^{8}}=\frac{13+i}{15} \\
& c^{\prime}=\frac{-13-9 i}{15}, \quad d^{\prime}=\frac{-8-14 i}{15}, \quad e^{\prime}=\frac{-13-14 i}{15} \quad \text { et } \quad d^{\prime}=\frac{7-14 i}{15}
\end{aligned}
$$

Remarque. Nous retrouvons d'une autre façon un résultat de Benedek et Panzone (voir [3]). Ce qui est nouveau avec notre méthode est qu'elle permet de trouver tous les points strictement extrémaux du fractal du dragon.

8. Généralisation aux $k$-fractals du dragon. Les $k$-fractals $d u$ dragon $(k \geq 1)$ sont les ensembles $\mathcal{D}_{k}=\left\{\sum_{n=1}^{\infty} a_{n} /(-k+i)^{n} \mid a_{n} \in\left\{0,1, \ldots, k^{2}\right\}\right\}$. Ce sont des compacts de $\mathbb{C}$ à frontière fractale (voir [6], [7], [12], [13]).

De la même manière que le fractal de Rauzy, nous pouvons déterminer les points strictement extrémaux de $\mathcal{D}_{k}, k>1$. Il suffit de coder par

$$
a_{n}= \begin{cases}k^{2} & \text { si } \cos (n \theta-a)>0 \\ 0 & \text { si } \cos (n \theta-a)<0 \\ \text { arbitraire } & \text { si } \cos (n \theta-a)=0\end{cases}
$$

où $\theta=\arg (1 /(-k+i))$. Le théorème (classique) qui va suivre montre que nous sommes dans la même situation que celle du fractal de Rauzy.

ThÉORÈme 8. Pour tout $k>1, \arg (1 /(-k+i)) / \pi \in \mathbb{R}-\mathbb{Q}$.

Preuve. Supposons que $\theta /(2 \pi)$ est rationnel. Il est clair que $\cos (\theta)$ est un nombre algébrique de degré au plus deux. Comme $[\mathbb{Q}(\cos (\theta)): \mathbb{Q}]=\psi(n) / 2$ où 
$n$ est le dénominateur de $\cos (\theta)$ et $\psi$ est la fonction d'Euler, nous déduisons que $\psi(n) \leq 4$, ou encore $n$ peut prendre seulement les valeurs $1,2,3,4,5,6,8,10,12$. Une simple vérification montre que ce n'est pas le cas.

Remarque. Notre méthode de la construction de l'enveloppe convexe se généralise aux ensembles de la forme $\left\{\sum_{i=0}^{\infty} a_{i} \gamma^{i} \mid\left(a_{i}\right) \in A^{\mathbb{N}}\right\}$ où $A$ est un ensemble fini de réels positifs contenant 0 , et $\gamma$ un nombre complexe de module $<1$.

Remerciement. L'auteur remercie le rapporteur pour ses remarques intéressantes et de lui avoir indiqué une démonstration assez simple du théorème 8 .

\section{Références}

[1] P. Arnoux, Un exemple de semi-conjugaison entre un échange d'intervalles et une rotation sur le tore, Bull. Soc. Math. France 116 (1988), 489-500.

[2] M. Barnsley, Fractals Everywhere, Academic Press, 1988.

[3] A. Benedek and R. Panzone, The set of Gaussian fractions, in: Proc. Second Conf. Math. "Dr. Antonio A. R. Monteiro" (Bahia Blanca, 1993), 11-40.

[4] M. Berger, Géométrie 2, Cedic/Nathan, 1977.

[5] F. M. Dekking, Recurrent sets, Adv. Math. 44 (1982), 78-104.

[6] W. J. Gilbert, Complex numbers with three radix expansions, Canad. J. Math. 34 (1982), 1335-1348.

[7] _ - Fractal geometry derived from complex bases, Math. Intelligencer 4 (1982), 7886.

[8] - Fractal dimension of sets derived from complex bases, Canad. Math. Bull. 29 (1986), 495-500.

[9] J. E. Hutchinson, Fractals and self similarity, Indiana Univ. Math. J. 5 (1981), 713-747.

[10] S. Ito and M. Kimura, On the Rauzy fractal, Japan J. Indust. Appl. Math. 8 (1991), 461-486.

[11] S. Ito and M. Mizutani, Potato exchange transformations with fractal domains, preprint.

[12] J. Kátai and J. Szabó, Canonical number systems for complex integers, Acta Sci. Math. (Szeged) 37 (1975), 255-260.

[13] D. E. Knuth, The Art of Computer Programming, Vol. 2, Seminumerical Algorithms, Addison-Wesley, Reading, MA, 1981.

[14] O. Lehto, Univalent Functions and Teichmüller Spaces, Springer, 1986.

[15] A. Messaoudi, Autour du fractal de Rauzy, Thèse de l'Université de la Méditérannée, Aix-Marseille II, 1996.

[16] - Propriétés arithmétiques et dynamiques du fractal de Rauzy, J. Théor. Nombres Bordeaux 10 (1998), 135-162.

[17] G. Rauzy, Nombres algébriques et substitutions, Bull. Soc. Math. France 110 (1982), 147-178.

[18] G. Rote, Sequences with subword complexity 2n, J. Number Theory 46 (1972), 196-213. 
[19] V. Sirvent, Properties of geometrical realisations of substitutions associated to a family of Pisot numbers, Ph.D. thesis, Univ. of Warwick, 1993.

[20] W. P. Thurston, Groups, tilings, and finite state automata, AMS Colloquium lectures, 1990.

Institut de Mathématiques de Luminy

CNRS-UPR 9016

163, avenue de Luminy Case 930

13288 Marseille Cedex 9, France

E-mail: messaoud@iml.univ-mrs.fr 